\title{
PETRARCA GHIBELLINO? ROMA E L'IMPERO NEI RERUM FAMILIARIUM LIBRI
}

\author{
MARIO CONETTI (*)
}

Nota presentata dal s.c. Gianmarco Gaspari

(Adunanza dell'8 giugno 2017)

SunTO. - Alcuni brani delle Familiari di Petrarca, non molto numerosi ma di grande importanza, affrontano il tema delle istituzioni imperiali in relazione particolarmente alle tensioni che segnano la politica italiana. Petrarca non elabora un pensiero politico istituzionale ben definito, pure l'idea imperiale di Roma è centrale. Appare possibile dimostrare che Petrarca risente l'influsso dei documenti ufficiali di Arrigo VII, in parte forse di Manfredi di Svevia, ma soprattutto della civilistica e delle fonti giuridiche romano giustinianee. Questi recuperi si inseriscono probabilmente dentro a un progetto culturale volto a restituire nel presente, almeno in parte, la grandezza della civiltà romana antica. Entro tale impegno però i temi politici e giuridici hanno un ruolo strumentale a esigenze dei centri di potere cui Petrarca è di volta in volta legato, in senso lato ghibellini, in particolare i Visconti ma anche lo stesso imperatore Carlo IV. Abbiamo così Petrarca che esalta in modo convinto la tradizione romana, anche quella imperiale; ma si mostra ghibellino di occasione, più per esigenze contingenti di comunicazione e di linea strategica dei centri politici cui è legato.

$* * *$

ABSTRACT. - A few, but very meaningful pieces from Petrarch's Familiari deal with the Holy Roman Empire and its institutions, especially because of the role they played in italian politics. Although Petrarch is not a systematic political thinker, the imperial idea of Rome plays a pivotal role. It seems possible to demonstrare that Petrarch has been influenced by official documents by Henry VII, eventually Manfred of Suabia, and mostly by civil lawyers and the sources of Roman law. These last item belongs to Petrarch's commitment towards a recovery for his present days of Roman classical heritage. All this said, political issues still play only an instrumental role, connected with the immediate needs of those powers, the Visconti household first and most but also

(") Dipartimento di Diritto economia e culture, Università degli Studi dell'Insubria, Varese, Italia. E-mail: mario.conetti@uninsubria.it 
emperor Charles IV himself, Petrarch was intimately connected to. Though Petrarch sincerely advocates Roman classical tradition, he is a ghibellino only for a matter of opportunity, or rather of the opportunity of those powers he decided to serve, and their immediate political needs.

Alcuni brani, forse non molto numerosi ma evocativi, del grande epistolario di Petrarca si soffermano sull'impero, nella dimensione politica e istituzionale e nel legame inscindibile con l'Italia e in particolare con Roma.

È auspicio di chi scrive fare emergere le ragioni, le vicende contingenti e concrete, che possono avere determinato le varie prese di posizione. Rilevano in particolare i legami con altri testi e contesti, per suggerire da dove Petrarca può avere tratto le idee se non il linguaggio con cui parlare dell'impero di Roma. In questa prospettiva si segnala all'attenzione Arrigo VII di Lussemburgo: sia la sua figura e la sua politica quale si manifesta nei documenti usciti dalla sua cancelleria durante il periodo italiano, sia la memoria del suo programma di restaurazione imperiale. Appare ancora attiva la reminiscenza di un analogo sogno imperiale, quello di Manfredi di Svevia. Non sarà poi da trascurare la presenza del diritto civile.

Chi scrive non pretende di potersi misurare con tanta e tanto illustre tradizione di studi, che tocca il punto maggiore nell'opera miliare di Ernest Hatch Wilkins ${ }^{2}$, fondata sul principio fondamentale del transito dal testo alla biografia. Principio che, seppure con modalità diverse e in termini dubitativi e critici, si ritrova in un altro classico degli studi petrarcheschi: "Si la fuente primaria para la biografia son los escritos petrarquescos, que llegaremos a conocer con una discreta firmeza? La vida o bien la obra de Petrarca? Dichtung oder

1 Si tratta di temi e riferimenti che non è stato possibile rinvenire valorizzati in altri e precedenti studi critici. L'immagine di 'Petrarca ghibellino' è stata proposta in anni molto lontani (e in seguito mai più davvero ripresa almeno per quanto è dato sapere a chi scrive) da Bonaventura Zumbini (Studi sul Petrarca, Napoli, 1878, pp. 175-265) che lo rappresenta come un "Farinata magnanimo", animato da una incrollabile fede nella pars imperii secondo un modello ideale che risente appunto moltissimo della matrice dantesca. La proposta interpretativa di Zumbini è basata su una analisi e una ricostruzione logica, se non propriamente sistematica, degli scritti petrarcheschi, e non su ricerche quanto alla loro intertestualità.

2 Life of Petrarch, Chicago 1961; tr. it. di R. Ceserani Vita del Petrarca, Milano, 1964; riedizione a c. di L.C. Rossi, Milano, 2003. 
Wahrheit?"3. In anni molto prossimi questo principio appare ancora attivo, per cui Ugo Dotti ancorchè nel presentare un ritratto intenzionalmente "rivoluzionario" di Petrarca ha potuto ipotizzare che "l'opera... fa quasi tutt'uno con la vita, questa riflettendosi in quella" così lo studio esaustivo di Roberta Antognini sulle Familiari propone la lettura dell'epistolario nei termini di una autobiografia ${ }^{5}$.

Le pagine che seguono vogliono essere una lettura di testi e del loro significato politico, il che implica anche il tentativo di cercarne una ragione nella situazione contingente e di indicarne i riferimenti nell'intertestualità. Non intendono invece provare a ricostruire dal testo letterario la dimensione biografica dell'autore.

Non si troverà qui nemmeno uno studio di insieme sul pensiero politico di Petrarca e sulla sua visione del potere imperiale. Una simile ricostruzione presupporrebbe, nell'oggetto, se non la dimensione teorica di un sistema dottrinale, almeno il tentativo di tradurre i fatti politici in un sistema di valori ${ }^{6}$. Orbene, non pare possibile ritrovare questa dimensione e questo tentativo nelle pagine di Petrarca. Per quanto questa affermazione possa suonare perentoria, chi scrive è convinto che le notazioni sull'impero di Roma non si mostrino passibili di essere lette quali frammenti di una teoria. Sebbene tali notazioni siano organizzate a comporsi nell'ambito di un macrotesto (i ventiquattro libri de rebus familiaribus), rimangono pur sempre incidentali, tutto considerato ognuna di esse appare più legata al singolo microtesto e alle circostanze in cui ha visto la luce, che agli altri microtesti in cui ricorrono temi analoghi e posizioni simili ${ }^{7}$. Si presentano insomma quali prese di

\footnotetext{
3 F. Rico, Vida u obra de Petrarca, Padova - Chapel Hill, 1974, p. xvii.

4 U. Dotti, Petrarca civile. Alle origini dell'intellettuale moderno, Roma, 2001, p. 11. 2008.

5 R. Antognini, Il progetto autobiografico delle Familiares di Petrarca, Milano,

6 Chi scrive ritiene che questo schema rappresenti il magistero di Carlo Dolcini nella storia del pensiero politico medievale; cfr. ad es. le considerazioni su Bartolo da Sassoferrato, "impegnato a trasferire... nel linguaggi della tecnica giuridica la coscienza etico-politica della divisione d'Italia" (C. Dolcini, I giuristi medievali tra assolutismo e costituzionalismo, in Il pensiero politico. Idee teorie dottrine, I, a c. di C. Dolcini, Torino, 1999, pp. 121-43, a p. 125).

7 Tali considerazioni, è tanto ovvio che non sarebbe nemmeno il caso di precisarlo, non valgono per tutti i temi sviluppati nei Rerum familiarium libri e nemmeno per la dimensione di impegno civile, il che esulerebbe dall'ambito intenzionalmente molto concentrato del presente contributo e andrebbe a inficiare tutto un filone di
} 
posizione magari enfatiche ma non sostenute da un impianto ideale e concettuale.

Si avvertirà qui la presenza della risalente intuizione complessiva di Francesco De Sanctis, per cui "la politica fu per il Petrarca non vocazione, ma occasione" 8 , e "secondo il vento, ora ti parla della guerra santa contro gl'infedeli, ora della Repubblica romana, ora della cacciata de' barbari, ora della Chiesa di Roma". Del resto, impostazioni critiche diverse sono riuscite a rivendicare una coerenza di visioni politiche a Petrarca ma non una sistematicità di riflessione teorica; è il caso di Giuseppe Brizzolara, che ricostruisce un nucleo politico ideale che "non varia secondo il variare degli eventi" ${ }^{10}$, ma non per questo può venire presentato quale dottrina compiutamente elaborata. Anche uno studio monografico che indaga con grande acribia le idee politiche di Petrarca, come quello di Rodolfo De Mattei ${ }^{11}$, recuperando sul piano delle idealità una profonda coerenza dovuta alla matrice classica (ciceroniana e stoica) e cristiana, o meglio alla loro consapevole fusione, parla fin dal titolo di un "sentimento politico" e non di un "pensiero". Forse solo Nicola Zingarelli ha potuto parlare di un "sistema politico" petrarchesco, ravvisandone la cifra ideale nella sintesi di repubblica e impero, secondo una impostazione dottrinale che risente delle categorie elaborate da Marsilio da Padova, in particolare l'identificare la sede della sovranità nel "legislator humanus", che è per eccellenza il popolo ${ }^{12}$.

La scelta di concentrarsi sui Rerum familiarium libri ${ }^{13}$ è fondata sulla consapevolezza della "netta divaricazione delle due esperienze sti-

ricerca che ha ricostruito le scelte strategiche per cui Petrarca ha ordinato le lettere e i libri per mettere in particolare risalto scelte e episodi (si pensi da ultimo e in particolare a U. Dotti, Petrarca cit.). Vanno riferite esclusivamente al tema e ai testi qui oggetto di indagine.

8 F. De Sanctis, Saggio critico sul Petrarca, Napoli, 1883, p.164.

9 Ibidem.

10 G. Brizzolara, Il Petrarca e Cola di Rienzo, s. 1., 1899, p. 247.

11 R. De Mattei, Il sentimento politico del Petrarca, Firenze, 1944.

12 N. Zingarelli, Il Petrarca, s. l. né d. ma 1928, p. 415 in partic. Il suggerimento quanto alle parentele con Marsilio è affascinante e forse meriterebbe tenere conto, più di quanto abbia fatto la critica sino a ora, verso altri contesti tematici; ma non pare fruttuoso rispetto al tema dell'impero e di Roma.

13 Si segue qui intenzionalmente la scelta di Giuseppe Billanovich quanto al titolo da conferire al grande epistolario. Per le alternative e i significati di cui ogni scelta è carica cfr. Antognini, Il progetto cit., pp. 18-20. 
listiche, quella latina e quella volgare" ${ }^{14}$, ove è il latino a essere "lingua di comunicazione e pratica" ${ }^{15}$. Per cui risulta opportuno volgersi, in via non esclusiva ma certo previa, ai testi in latino nella ricerca di quelle idee politiche che sono per l'appunto intimamente legate sia alle esigenze che emergono dalla prassi sia a una necessità di comunicazione, seppure dettata dalle relazioni diplomatiche, come appare in modo particolare proprio nella sezione maggiore dell'epistolario di Petrarca ${ }^{16}$. Inoltre, se l'insieme delle lettere familiari costituisce, secondo una recente ricostruzione globale, "ritratto di sé e critica di sé (e dei tempi)" 17 , per cui "artefice geniale, Petrarca trova il modo di rendere l'incertezza e la frammentarietà della vita... combinando due generi multiformi, aperti alla sperimentazione: la scrittura epistolare, genere antico con una lunga tradizione, e l'autobiografia, genere che nel Medioevo era ancora tutto da inventare"18; allora proprio un percorso nei Rerum familiarium libri potrà permettere di cogliere squarci notevoli, non si vuole dire delle convinzioni politiche di Petrarca, ma del modo in cui, scendendo a patti con le necessità rappresentategli dai poteri cui di volta in volta era legato, ha voluto fornire qualche visuale della vita associata e degli assetti di potere ${ }^{19}$.

\section{AMATOR IMPERII}

Il percorso può avviarsi col momento più tipico di Petrarca letterato: l'autobiografia, la riflessione su se stesso di cui sovente si compiace nei Familiarium rerum libri. Si veda a tale proposito la Fam. XXIII

14 M. Martelli, Petrarca: psicologia e stile, in Petrarca, Opere, I, Firenze, 1975, pp. xiii-xlvii, p. xiii.

15 Ivi, p. xiv.

16 La scelta di concentrare l'attenzione sulle Familiari esime dal prendere in considerazione la questione, che potrebbe risultare qui tematicamente significativa, dei rapporti tra Petrarca e Cola di Rienzo; la presenza non tanto di Cola quanto in particolare del suo programma volto a restaurare i fasti di Roma è trascurabile, si ritrova invece nelle Variae e nelle Sine nomine. Del resto sul tema del nesso tra Petrarca e Cola di Rienzo si può rimandare alle considerazioni di G. Baldassarri, op. cit. infra a n. 19, partic. pp. 70-6, e a G. Ferraù, Petrarca, la politica, la storia, Messina. 2006.

17 Dotti, Petrarca cit., p. 32.

18 Antognini, Il progetto cit. p. 12.

19 Si tratta di una scelta complementare a quella che ispira un recente studio, non lontano per oggetto e temi: G. Baldassarri, Unum in locum. Strategie macrotestuali nel Petrarca politico, Milano, 2009. 
$2^{20}$, scritta all'imperatore Carlo IV da Milano e datata al 21 marzo, per comune consenso fondato sull'evidenza interna, del $1361^{21}$. Riprende alcuni temi e contenuti della lettera che aveva inaugurata la corrispondenza con l'imperatore della casa di Lussemburgo, oltre un decennio prima, e che gli pare tuttora attuale. Opportunamente presenta un ricordo di quel momento ${ }^{22}$. Il Petrarca che ormai può vantare una familiarità con l'imperatore, riflette sul Petrarca di oltre un decennio prima e di un momento molto significativo, in cui per la prima volta aveva avuto l'ardire di rivolgere la penna all'augusto corrispondente. Dichiara adesso di averlo fatto mosso dalla sola consapevolezza di essere "amator imperii”. Consapevolezza che, a quanto pare, non solo non lo abbandona, ma si è consolidata lungo gli anni.

Petrarca vuole descrivere una convinzione e un atteggiamento fondamentali. Lo fa con un sostantivo, "amator", che potrebbe rappresentare uno dei non frequenti casi in cui viene superata la divaricazione delle due esperienze letterarie e stilistiche, latina e volgare.

"Amator" e il sintagma col genitivo oggettivo sono schiettamente ciceroniani: pertanto si inscrivono in pieno nel progetto letterario latino di Petrarca. Nel latino di Cicerone stanno a indicare un rapporto di coinvolgimento profondo, sentimentale ma ancora più esistenziale, sul modello di un rapporto di "amicitia" ma con intensità e ampiezza maggiori, soprattutto alludendo a una condizione costante e non mutevole ${ }^{23}$. Il termine e il costrutto vengono ripresi da Agostino di Ippona ${ }^{24}$ e in una occasione Petrarca cita uno di questi passi agosti-

20 Il testo dei Rerum familiarium libri qui utilizzato è quello dell'edizione critica data da V. Rossi e U. Bosco per l'Edizione nazionale delle opere di Francesco Petrarca, X-XIII, Firenze, 1933-1942; come riportato nell'edizione Petrarca, Opere, cit.

21 Il carteggio tra Petrarca, l'augusto corrispondente e i personaggi legati alla sua corte è raccolto, tradotto e commentato in F. Petrarca, Lettere all'imperatore. Carteggio con la corte imperiale di Praga (1351-1364), a c. di U. Dotti, Parma, 2008.

22 Fam. XXIII 2, 10: "Undecimus, nisi fallor, annus agitur ex quo primum moras tuas increpui, homo tunc incognitus tibi sed et nosci cupiens et amator imperii; et si libertatem meam tunc non tantum tulisti sed laudasti, certe, iam senioribus, et michi plus libertatis et tibi excusationis aliquanto est minus. Relege que tunc scripsi. Videbis quanto tibi nunc singula convenientius dici possint, eo quod et occasio ingens pretermittitur et plus vite abiit, minus restat".

23 Cfr. ad es. Cic., Ad Att. 10, 2: "amator pacis"; Ad Att., 1, 14: "Messalla consul est... nostri laudator, amator, imitator"; Id., Tusc. 12, 27: "ut inter ebrietatem et ebriositatem interest aliudque est amatorem esse, aliud amantem".

24 Ad es. cfr. Aug., De civitate Dei, 8, 1, 3: "verus philosophus est amator Dei"; 
niani, manifestando così di rifarsi tanto al modello letterario quanto a quello ideale e autobiografico. Un esempio di ripresa più risalente di tali modelli, nel latino quale lingua anche politica, si ha con Giovanni di Salisbury ${ }^{25}$.

Allo stesso tempo, "amator" non può non presentarsi come un calco linguistico sul volgare del poeta di Laura, con la ricchezza di significati e sfumature. Da questo corto cirtuito tra latino e volgare emerge un coinvolgimento esistenziale e complessivo verso l'oggetto dell'amore. L'"imperium" appunto, dove ancora una volta si confondono antichità e attualità, con una corrispondenza alla scelta del termine "amator" che è del linguaggio di Petrarca novello Cicerone quanto di Petrarca poeta in volgare. In un momento importante di riflessione su se stesso, Petrarca sceglie di autodefinirsi coinvolto in un rapporto di "amor" con l'impero.

È questo l'unico modo con cui il poeta può corrispondere a quei sentimenti di benevolenza o addirittura di affetto che l'imperatore gli ha dimostrato e continua a mostrargli, come afferma nell'esordio della stessa missiva ${ }^{26}$. La scelta di dedicare la sezione di apertura, quasi un lungo prologo, della lettera a ricordare la considerazione positiva di Carlo IV nei suoi confronti, richiede cioè, quale necessario corrispettivo retorico, di presentare da parte sua un sentimento ancora più intenso nei confronti e di chi deteneva il potere imperiale, e dell'impero stesso. Il momento autobiografico con l'affermazione di un coinvolgimento si potrebbe dire esistenziale prima ancora che politico nei confronti dell'impero va allora compreso nella prospettiva di una comunicazione letteraria e soprattutto diplomatica, viste le circostanze in cui è stata composta la Fam. XXIII $2^{27}$.

questo passo viene citato esplicitamente da Petrarca, Contra medicum quendam, 2. Cfr. inoltre De civitate Dei, 14, 6, 9: "homo, qui secundum Deum non secundum hominem vivit, oportet ut sit amator boni"; Epistulae, 110, 6, 2: "bonus es amator iustitiae".

25 Iohannis Sarisberiensis Policraticus, 4, 11 (PL 199, 661): "philosophus Dei amator est"; Policraticus 3, 14 (PL 199, 506): "aut virtutis amator est, aut iuris constitutioni obtemperans".

26 Fam. XXIII 2, 1: "maiestati cesaree carum esse fore", per una sintesi di quanto sviluppato nei primi 8 paragrafi, tutti dedicati appunto alle manifestazioni della benignità e del memore pensiero di Carlo IV verso Petrarca.

27 Per cui cfr. infra pp.18-9. 


\section{LA TRADIZIONE GHIBELLINA DI ARRIGO VII (E DI MANFREDI DI SVEVIA)}

La prima delle tredici familiari che Petrarca rivolge all'imperato$\mathrm{re}^{28}$, citata nel paragrafo precedente, è un testo celebre, dove in modo esemplare si fondono la retorica carica di riferimenti classici e la contingenza politica più spicciola. Era stata infatti dettata dalle esigenze del cardinale Guy de Boulogne e scritta presumibilmente molto in fretta: infatti è datata al 24 febbraio e il cardinale era giunto a Padova, dove si trovava Petrarca, il 4 febbraio 1350 per la traslazione delle reliquie di S. Antonio. Lì era stato coinvolto nella contesa tra il Patriarca d'Aquileia, Bertrand de Saint Geniès, e il Conte di Gorizia. Non era riuscito a risolvere il problema politico e territoriale, anzi si era convinto che solo un intervento diretto imperiale avrebbe potuto garantire la pace di quell'area e soprattutto i diritti del principato ecclesiastico aquileiese ${ }^{29}$. Del resto, un decennio prima Carlo di Lussemburgo aveva già avuto modo di impegnarsi direttamente a difesa degli interessi del patriarcato sempre nei confronti di minacce espansionistiche provenienti dai dinasti goriziani ${ }^{30}$. Nulla di meglio per il principe francese della chiesa, nell'impegno a convincere Carlo IV a varcare le Alpi, di valersi anche della penna di Petrarca che in quel momento era a portata di mano.

La necessità dettata dalla contingenza politica richiedeva un intervento energico e efficace sul piano letterario. Petrarca, in questa chiave, sceglie di chiudere la prima lettera con la prosopopea di Arrigo VII di Lussemburgo, nonno di Carlo IV; che, secondo una figura elaborata, si inserisce dentro a un'altra prosopopea, quella di Roma che occupa grosso modo la seconda metà della lettera. Evocare la figura dell'illustrissimo avo e cedergli direttamente la parola doveva certo, e senza

28 Per una visione di insieme della corrispondenza cfr. P. Piur, Petrarcas Briefwechsel mit deutschen Zeitgenossen, Berlin, 1933; P. Lukesova, Le lettere di Francesco Petrarca all'imperatore Carlo IV. Bakalarska diplomova pra e, Mazarykova Univerzita, Filozoficka Fakulta, 2008.

29 Cfr. P. Jugie, L'activité diplomatique du cardinal Gui de Boulogne au milieu du XIVe siècle, "Bibliothèque de l'Ecole des Chartes 145 (1987), pp. 99-127; G. Brunettin, Bertrando di Saint-Geniès Patriarca d'Aquileia, Spoleto, 2004.

30 Le opere di riferimento su Carlo IV sono pur sempre G. Biermann, Karl IV. römisch-deutsche Kaiser und König von Böbmen, Wien 1878; E. Werunsky, Geschichte Kaiser Karls IV. und seiner Zeit, Innsbruck 1880-92; T. Mentzel, Italienische Politik. Kaiser Karls IV., Blackenburg, 1885. 
troppa difficoltà, presentarsi a Petrarca quale espediente efficace per muovere Carlo IV all'azione. Va però osservato con attenzione come il modo in cui viene presentata la figura di Arrigo VII e le parole che Petrarca gli mette in bocca escono da quelli che potevano essere riferimenti vaghi e generici dettati dalle esigenze retoriche e si sostanziano invece di temi e rinvii ben definiti ${ }^{31}$.

Al $\$ 25$ l'iterazione del termine "unus" non dovrebbe avere lo scopo di sottolineare l'inutilità di appesantire il testo con molteplici riferimenti, quando è sufficiente uno solo purchè davvero significativo; quanto piuttosto di porre in evidenza la singolarità di Arrigo VII, della sua esperienza storico politica. Si tratta di una ipotesi di lettura, dato che esplicitare le intenzioni contenutistiche nelle scelte di stile di Petrarca è impresa tutt'altro che agevole. È unico, Arrigo VII, perché è il solo imperatore che si sia presentato in tempi recenti nella pienezza del potere imperiale. Rammenta qui l'impatto fortissimo che Arrigo VII ebbe in Italia, l'eco subito suscitata nella pubblicistica (fin troppo scontato il riferimento a Dante o a Cino da Pistoia).

Nello stesso giro di frase in cui presenta l'assoluta singolarità di Arrigo VII e della sua politica, lo caratterizza anche come "non procul", "non ex annalibus querendus". Il riferimento chiaro è al rapporto di parentela; l'intento più profondo consiste nell'avvicinare e il personaggio e la sua esperienza politica a Carlo IV, che potrebbe esserne l'erede in più di un senso.

Il dramma di Arrigo VII, la conclusione tragica e repentina della

31 Fam. X 1, 25: "Unus tibi, non procul, non ex annalibus querendus, unus tibi pro omnibus satis erit: Henricus, eterne memorie serenissimus avus tuus, cui si ad explenda que sacra mente conceperat, vite spatium suffecisset, versa rerum sorte et afflictos hostes et me regnantem et liberrimos Italie populos ac felicissimos reliquisset. Spectat ille nunc celorum perpes incola, dies computat horasque dinumerat teque mecum increpitans alloquitur: 26 Nepos amantissime, quo superstite nec bonorum spes nec ego totius interii, Romam nostram atque illius lacrimas precesque dignissimas amplectere reformandeque reipublice propositum, quod mea mors, mundo quam michi damnosior, prevertit, et inefficacem animi mei zelum pari ardore prosequere, sed felicius sed letius. Incipe, ne moreris, et nostri memor scito te esse mortalem. 27 I celer et gaudentia alpium claustra transcende; Roma sponsum, sospiratorem suum vocat Italia et tuis pedibus tangi cupit; expectant te leti colles ac flumina, expectant urbes et oppida, expectant bonorum agmina; et si te nil aliud urgeret nisi quod malis nunquam satis distulisse, bonis nunquam satis festinasse videberis, sat cause est ut his gaudium, illis penam vel si resipiscere maluerint, veniam laturus acceleres. Solus enim es cui Deus omnipotens interrupti consilii mei dilatam gloriam reservavit”. 
vita e dell'azione politica, vengono enfatizzati con una duplice funzione, letteraria e politica. Sul piano letterario, tale enfasi serve a commuovere. Sul piano politico, ha lo scopo di mostrare come quel programma non fosse irrealizzabile: se è fallito, ciò non si deve a difficoltà intrinseche, ma a un colpo di sfortuna. Pertanto, può essere ripreso e portato a termine con successo.

Il discorso arriva così al culmine e al punto centrale, la comprensione della politica di Arrigo VII. Petrarca ne riassume così il programma: l'annientamento dei nemici del potere imperiale; la libertà e la felicità per i popoli d'Italia.

La repressione nei confronti delle opposizioni ostinate al potere imperiale è stata senza dubbio un elemento cruciale della politica di Arrigo VII. Dalle città comunali italiane a re Roberto d'Angiò la sua azione in Italia è costellata di interventi, prima di tutto appunto contro i comuni che dimostravano nei fatti di non volere riconoscere quella sovranità imperiale cui pure formalmente non potevano che dichiararsi sottoposti; dove l'impiego della forza, politica e militare, di cui poteva disporre si accompagna sempre alla giustificazione sul piano teorico, giuridico e amministrativo, degli interventi stessi ${ }^{32}$. Proprio il contenzioso col sovrano angioino e la relativa accusa di lesa maestà costituiscono l'occasione per un intervento normativo, la costituzione imperiale "Qui sint rebelles", dove si ha la definizione precisa della fattispecie criminosa di ribellione al potere imperiale ${ }^{33}$. Del resto, Arrigo stesso nell'enciclica maggiore promulgata in occasione dell'incoronazione imperiale, che è allo stesso tempo un programma politico e un denso trattato dottrinale de potestate imperatoris, ricorda come il suo primo intervento politico significativo in Italia dovette essere proprio volto a reprimere la ribellione di Brescia ${ }^{34}$. Individuare il primo punto fondamentale del

32 Cfr. ad es. i bandi dell'aprile 1312 contro alcune città lombarde e toscane: Monumenta Germaniae Historica, Constitutiones et acta publica imperatorum et regum (d'ora in poi MGH Const.), vol. 4 t. 2, nrr. 768 e 769, pp. 757-64.

33 Del 2 aprile 1313, cfr. MGH Const. 4/2, nr. 931, p. 966. Sulle costituzioni pisane cfr. E. Betti, La dottrina costruita da Bartolo sulla constitutio Ad reprimendum, in Bartolo da Sassoferrato. Studi e documenti per il VI centenario, II, Milano, 1962, pp. 37. 47; D. Quaglioni, 'Fidelitas babet duas habenas'. Il fondamento dell'obbligazione politica nelle glosse di Bartolo alle costituzioni pisane di Enrico VII, in Origini dello Stato. Processi di formazione statale in Italia tra medioevo e età moderna, a c. di G. Chittolini, A. Molho, P. Schiera, Bologna, 1994, pp. 381-96.

34 MGH Const. 4/2, nr. 801, pp. 801-4, p. 803: "Quia vero dum per Lombardiam 
programma politico di Arrigo VII nella repressione degli avversari del potere imperiale, significa avere di questo programma, quale poteva essere desunto dai documenti pubblici, una conoscenza per lo meno discreta.

Il crescendo culmina nel rendere gli italiani "liberrimos" e "felicissimos". Il centro del programma politico di Arrigo VII viene espresso da Petrarca nei termini di assicurare libertas e felicitas per l'Italia. In questo caso, a differenza del punto precedente, non pare possibile individuare nella documentazione ufficiale di Arrigo VII un antecedente.-

Nei documenti pubblici di Arrigo VII il termine concetto "libertas" non ricorre frequentemente, e quando è presente viene per lo più utilizzato al plurale a indicare le prerogative imperiali ${ }^{35}$; il sostantivo "liber" e altri termini con la medesima radice sono praticamente assenti. "Felicis" e forme derivate non ricorre mai se non nelle formule stereotipate cancelleresche. Appare pertanto necessario volgersi a altri antecedenti testuali.

Si potrebbe invece sentire qui un'eco del manifesto di Manfredi di Svevia ai romani, commovente affermazione della missione imperiale di Roma nel maggio 1265, dove il primato politico dell'Urbe è in sé "felix" per definizione ${ }^{36}$. Se il riferimento, per quanto indiretto, fosse

terram nostro imperio subiectam contenderemus ad Urbem, ut in die assumptionis prefate de coronationis nostre felicibus auspiciis gauderemus, civitas Brixiensis nefando ausu nostro culmimi rebellavit, et indignum erat minusque providum, nos inde discedere, donec sub sceptro nostro colla submitteret, ne forte fieret reliquis Lombardie civitatibus in scandalum et ruinam, ad tempus negocium coronationis predicte suspendimus et dictam civitatem tam diu dura obsidione vexavimus, donec non valens diucius potencie nostre resistere, se libere nostris manibus reddidit et clemencie nostre arbitrio de commisso in magestatem nostram crimine castigandam puniendamque submisit".

35 Cfr. ad es. MGH Const. 4/2, nr. 916, p. 933.

36 Manfredi di Svevia, Epistola ad Romanos (24 maggio 1265), ed. in E. Dupré Theseider, L'idea imperiale di Roma nella tradizione del Medioevo, pp. 216-29, \$3, p. 217: "Et quia tocius nostre intencionis versabatur intencio et alcius nunc versatur, ut per nostre potencie apices imperialis celsitudinis promoveatur fastigii meritum, ut mundi fluctuantis exposcit necessitas, ac felix genus reipublice romanorum singulis preponatur orbis nacionibus, ut legum demandat auctoritas". Ibidem, $\mathbb{S} 11$, p. 221 : "felicissima urbs Roma, mundi ac imperii capud". Cfr. inoltre A. Frugoni, Il manifesto di Manfredi ai Romani, Palermo, 1951 (ora in Id., Scritti su Manfredi, Roma 2006, pp. 45-82); M. Brantl, Urkunden - und Kanzleiwesen Manfreds von Sizilien 1250-1266, Archiv für Diplomatik 51 (2005), pp. 127-252. Sul codice, testimone unico che ce l'ha trasmessa, cfr. per lo meno C. Villa, Raccolte documentarie e ambizioni storiografiche: il "progetto" del manoscritto Fitalia (Palermo, Biblioteca della Società Siciliana per la storia 
davvero presente e attivo, si avrebbe allora come la costruzione di una articolata genealogia ghibellina, che dall'ultimo campione del sogno imperiale svevo attraverso Arrigo VII può raggiungere Carlo IV.

Il punto d'arrivo della prosopopea di Arrigo VII è infatti questo: nessuno meglio del nipote può proseguire il suo cammino bruscamente interrotto. L'attesa di cui sarebbe circondata in Italia la figura di Carlo e che dal mondo ambiente fisico con un crescendo coinvolge le città per culminare nei "bonorum agmina" sente certo la memoria dell'accoglienza tributata a Arrigo VII in particolare dalle città ghibelline toscane e proprio da Arezzo.

Nelle urgenze di una situazione politica contingente, Petrarca si trova a comporre una lettera che possa contribuire a convincere l'imperatore a varcare le Alpi; allo stesso tempo, tale richiesta costituisce per lui una opportunità notevole per accreditarsi positivamente verso un fronte ghibellino, di cui il cardinale di Boulogne rappresentava in quel momento un esponente di spicco. I contatti con Luchino Visconti, punto di riferimento del ghibellinismo padano, erano iniziati da tempo, come dimostra la Fam. VII 15 a lui diretta e probabilmente del marzo $1348^{37}$; Petrarca poteva già stare pensando se non alla dimora milanese, per lo meno a stabilire rapporti più intensi con il casato della biscia ${ }^{38}$. La morte improvvisa di Luchino Visconti nel gennaio del 1349, seguita nel maggio dello stesso anno da quella di Paganino da Besozzo che certo rappresentava un tratto d'unione notevole, dovettero interrompere l'avvicinamento di Petrarca alla signoria milanese. A maggior ragione poteva in seguito volere cercare di accreditarsi positivamente di fronte al ghibellinismo di cui i Visconti rappresentavano il vertice politico e ideale. Fa ampio utilizzo della pas-

patria I B 25), in Confini dell'Umanesimo letterario. Studi in onore di Francesco Tateo, cur. M. de Nichelo, G. Distaso, A. Iurilli, Roma, 2003, pp. 1417-27; P. Colletta, Per un'edizione del Codice Fitalia: l'apporto della tradizione manoscritta della Cronica Sicilie, Archivio Normanno-Svevo 4 (2013-2014), pp. 103-24.

37 La missiva è datata da Petrarca al 13 marzo, Fracassetti completa con l'anno 1348. Si può seguire l'ipotesi di F. Novati che sia stato Paganino da Besozzo mentre si trovava a Parma, dove era stato inviato quale podestà dopo la conquista viscontea, a propiziare tra il 1347 e il '48 l'avvicinamento di Petrarca a Luchino (F. Novati, Il Petrarca ed $i$ Visconti. Nuove ricerche su documenti inediti, in Petrarca e la Lombardia, Milano, 1904, pp. 11-57, le pp. 13-6). Sui rapporti tra la produzione di Petrarca e le esigenze politiche dei regimi signorili cfr. G. Ferraù, Petrarca, la politica e la storia, cit. a n. 6.

38 Già G. Martellotti, Saggi petrarcheschi, Bologna, 1949, p. 123 parla dei “contatti ch'egli ebbe con ambienti ghibellini, ancor prima di prendere stanza a Milano”. 
sione per la classicità imperiale e della conoscenza accumulata in proposito. Accanto a questa però impiega anche riferimenti molto più prossimi, a una tradizione ghibellina legata ai documenti e alle figure forse di Manfredi di Svevia, certo di Arrigo VII di Lussemburgo. Visti i tempi stretti di confezione della Fam. X 1, doveva trattarsi di echi e riferimenti a lui già disponibili. Dovrebbe essere proprio il ghibellinismo toscano a costituire il polo d'attrazione verso Arrigo VII e Manfredi; oltre alla lunga familiarità con casa Colonna, dati i rapporti stretti che avevano legato a Arrigo VII Stefano Colonna il Vecchio ${ }^{39}$.

A ogni modo, importa rilevare come il "ghibellinismo" 40 di questa missiva sia strumentale, sia alle esigenze di relazioni diplomatiche da parte di chi l'aveva ispirata, sia alle strategie di affermazione personale di Petrarca stesso.

Va rilevata un'ulteriore consonanza notevole tra le lettere dirette a Carlo IV e i documenti pubblici di Arrigo VII. Nella lettera, la terza della serie, con cui Petrarca ribatte alla risposta, giuntagli tardivamente, dell'imperatore alla sua prima missiva ortatoria, pur nella durezza dei toni o piuttosto per mitigarla e onde possa venire letta nella giusta luce, si preoccupa a più riprese di chiarire quale sia il proprio rapporto nei confronti del sovrano, il suo atteggiamento vero e fondamentale. Lo fa ricorrendo al termine "fides" abbandonato: la libertà con cui ha espresso il suo pensiero e i "consilia" che potevano risultare sgraditi; per quanto forse manchevoli di "prudentia" sono sempre stati ispirati alla "fides" verso l'imperatore e vanno intesi quali manifestazioni di questa.

Orbene, "fides", meno spesso "fidelitas",-è il termine con cui nella documentazione pubblica di Arrigo VII ${ }^{42}$ viene caratterizzato il

39 La raccolta dei documenti pubblici di Arrigo VII (MGH Const. 4, ad indicem) conserva numerose e significative menzioni della presenza di Stefano. Cfr. H. Cochin, Note sur Stefano Colonna, Bullettin de la Société des antiquaires de la Morinie 7 (1887), pp. 1-28.

40 Per impiegare un termine sintetico a ricomprendere tutte le osservazioni formulate sopra.

41 Fam. XVIII 1, 3: "Consilium vero non amplectitur; malo michi fidem sine prudentia constare, quam sine fide prudentiam. 4 Dum tibi mea fides ac devotio probentur... 8 Si ergo tibi, Cesar, hoc consilium non placet, placeat fides ut facit”.

42 Impossibile indicare dei riferimenti, che sarebbero pressochè coestensivi alle raccolte stesse di tale documentazione. Per un esempio macroscopico cfr. proprio le costituzioni pisane di cui a n. 31 . 
rapporto dei sudditi verso il sovrano imperiale, per riassumere gli atteggiamenti anche pratici che devono tenere nei suoi confronti: un rapporto di sottomissione certo, ma pure di cooperazione pronta e aperta e sempre di abbandono fiducioso.

Certo, il termine concetto "fides" è un caposaldo per tanti linguaggi politici medievali e non è facile individuare un antecedente preciso. Pure, va tenuto presente come, soprattutto in tempi più prossimi a Petrarca, nessuno ne abbia fatto un uso tanto massiccio e sistematico quanto Arrigo VII con la sua cancelleria. Soprattutto, per quanto possibile ricostruire, Petrarca lo impiega nello stesso significato.

Si potrebbe vedere un'altra consonanza ancora tra le lettere familiari e i documenti di Arrigo VII. Il tema del ruolo provvidenziale di Roma e del suo impero universale, unificare l'orbe quale preparazione all'avvento del Cristo, redimendosi così almeno in parte dal segno negativo che, a partire da Babilonia, grava su tutti gli imperi, è a tal punto diffuso nella cultura medievale che non stupisce come nemmeno Petrarca si sia potuto o voluto esimere dal ripresentarlo anche in quella lettera dove spiega al suo Lelio il desiderio di risiedere per qualche tempo proprio a Roma ${ }^{43}$. Tale diffusione vastissima rende tanto più difficile individuare riferimenti testuali più prossimi e decisivi rispetto a quelli fondazionali e espliciti al De civitate Dei. Può non essere privo di significato segnalare la prossimità di toni, se non di contenuti, col già citato manifesto politico formulato da Arrigo VII in occasione della sua

43 Fam. XV 9 dove i $\$ \$ 4-6$ si diffondono sull'identificazione tra Babilonia e Roma in Agostino, per concludere al $\$ 7$ : "Verum enimvero hoc loco tacitum nullo modo vellem esse quod sequitur: 'per quam', inquit, 'Deo placuit orbem debellare terrarum et in unam sotietatem reipublice legumque perductum, longe lateque pacare'. Ecce audis almam urbem ut nomine Babilonis infamem, sic causa originis et dignatione precipua celestis providentie gloriosam. Poterat nempe per aliam, sed per hanc voluit, quam scilicet tanto operi pre cunctis ydoneam ab eterno previderat"; $\$ 9$; "Ecce quibus effectibus divina providentia romanum imperium fabricata est, ut esset scilicet mundo caput"; $\mathbb{1 5}$ : "Romam velut alteram Babilonem in occidente fundatam non infitior, ut scilicet iubente Deo imperium orientale, quod in Babilone multis seculis fuit, inde ablatum cum tempore pariter ad occasum iret, ubi Romam esse perspicuum sit; interesse autem quod et mores urbium diversi et de orientali non occidentale, sed universale imperium sit effectum omniumque sola verissima monarchia. 16 Quodsi propter totius domiti orbis in una urbe concursum et confusionem rerum maximam ac super omnia deorum turbam sacrorumque, Babilon hoc est confusio, dici potest, non obluctor neque contendo; sed audeo dicere illud sibi nomen excidisse quo primum die fallacibus diis exclusis, unius veri Dei cultum nomenque suscepit”. 
incoronazione imperiale ${ }^{44}$, dove il transito della monarchia universale a Roma costituisce il segno esteriore più notevole della preparazione alla salvezza. Prossimità che andrebbe a sostenere l'ipotesi di una conoscenza diretta da parte di Petrarca di un testo, l'enciclica appunto di Arrigo VII, che per sua natura doveva avere avuto una buona diffusione.

\section{ROMA E L'ITALiA SEDE DELL'IMPERO}

La prima lettera a Carlo IV delinea, certo allo scopo di suscitarne il coinvolgimento nelle vicende cisalpine, fin dalle battute iniziali un rapporto tra l'imperatore e l'Italia di vera appartenenza reciproca ${ }^{45}$. Nel prosieguo ${ }^{46}$ con lo svolgersi dell'argomentazione si ha un parallelo tra le due componenti fondamentali dell'impero medievale, Germania e Italia. Carlo IV può sì essere nato in Germania, ma in Italia è cresciuto e si è formato, prima di tutto si può pensare nelle qualità di capo militare e politico: con una allusione implicita ma chiara alle esperienze, sia quelle compiute al fianco del padre Giovanni di Boemia sia quelle vissute quale suo vicario ma in prima persona negli anni '30 del XIV secolo. Soprattutto, la Germania è solo una delle componenti dell'impero universale, un "regnum", sebbene di per sé importante e particolarmente significativo per Carlo. L'Italia invece è l'unica sede dell'“imperium”. Qui si ha il punto d'arrivo dell'argomentazione petrarchesca:

44 MGH Const. 4/2, nr. 801 (Litterae encylcicae imperatoris Iun. 29 1312, Encyclica in forma maiori), pp. 801-4, p. $802 \$ 1$ : "Et quamvis huiusmodi principatus prioribus seculis in diversis fuerit nationibus... novissime tamen appropinquante plenitudine temporis, quando idem Deus et dominus noster inenarrabili dignationis sue munificencia homo fieri voluit... dictum imperium transiit ad Romanos provide Dei disponente clemencia”.

45 Fam. X 1, 2: "Quid enim nostri et, si dici fas est, tui ipsius immemor factus es? quonam abiit Italie tue cura? Nos equidem sperabamus te, celitus nobis missum, libertatis nostre promptissimum assertorem; tu refugis et ubi facto opus est, longissimis consultationibus tempus trahis".

46 Fam. X 1, 6: "Pelle moras igitur, et quod grande aliquid aggressis utilissimum est, singulos dies magni extima; ea te cogitatio parcum temporis efficiet, ea te coget ut venias et inter adversitatum nostrarum nubila speratum nobis augustissime tue frontis lumen ostendas. 7 Non te transalpinarum sollicitudo rerum, non te natalis soli dulcedo retineat; quotiens Germaniam respexeris, Italiam cogita. Illic natus, hic nutritus; illic regnum, hic et regnum habes et imperium, ea quod nationum ac terrarum omnium pace dixerim, cum ubique membra, hic ipsum caput invenies monarchie". 
solo in Italia risiede il "caput monarchie". Le membra dell'impero universale si trovano ovunque, il capo è unico e può essere solo l'Italia; questo inerisce alla dimensione ontologica, all'essere stesso dell'impero, non può di conseguenza mutare ${ }^{47}$. Petrarca qui non si segnala certo per originalità: riprende una sorta di assioma di tutta la letteratura, in particolare giuridica e pubblicistica, sull'impero. Questa diffusione larghissima assieme alla rapidità dell'esposizione rende quasi impossibile individuare antecedenti precisi.

Come avviene per diversi temi sviluppati nella prima lettera della serie, anche questo si trova ripreso nella terza, semmai (ancora, come avviene sovente) in toni più incisivi e aspri ${ }^{48}$. L'avere sede in Italia inerisce alla dimensione ontologica dell'impero; proprio perché ne va dell'essenza stessa della monarchia universale, questo aspetto non è disponibile, non è modificabile da nessun intervento da parte degli esseri umani. Sembra di potere quasi leggere qui implicita una minaccia velata nei confronti di Carlo IV qualora pensasse di potere traslocare la sede dell'impero: il che è semplicemente impossibile, per lo meno comporterebbe l'annientamento dell'essere stesso dell'impero.

Lo stesso tema, esposto in modo tutto sommato analogo, ricorre a distanza di anni per quanto in circostanze non dissimili. La Fam. XXIII 2, datata 21 marzo (1361), è stata composta quando Petrarca era ormai insediato a Milano e funzionalmente agli interessi del potere visconteo, che in quelle particolari circostanze si sarebbe sentito garantire da una presenza imperiale attiva in Italia settentrionale ${ }^{49}$. Tra le

47 Per una visione della dimensione "italiana" nelle idee politiche di Petrarca cfr. A. Mazzocco, Un'idea politica italiana in Petrarca? in Petrarca politico. Atti del convegno (Roma - Arezzo, 19-20 marzo 2004), Roma, 2006, pp. 9-26.

48 Fam. XVIII 1, 32: "Tu si cuncta deliberas et in singulis immoraris, predicam tibi etsi forte non animo tuo gratum, et certe fidei mee debitum - falsus utinam sim aruspex - nullus erit rerum finis, alii ex aliis erumpent laquei, nunquam te Italia nunquam tu Italiam videbis, extra quam ubi imperii caput queras nescio".

49 Era quello un momento particolarmente teso nel confronto tra Bernabò Visconti in particolare e il cardinale Egidio Albornoz, indirettamente il papato. Nell'ottobre 1360 l'Albornoz era riuscito a strappare Bologna al controllo di Giovanni Visconti da Oleggio, ma ciononostante le ostilità proseguivano nelle vicinanze della città. Bernabò aveva compreso di non potere contare più su un intervento a lui favorevole di papa Innocenzo VI, che anzi sarebbe di lì a pochi mesi (maggio 1361) giunto a scomunicarlo; e si volgeva pertanto a cercare altri possibili e autorevoli appoggi, prima di tutto quello imperiale, al fine di non vedere definitivamente compromesso un momento rilevantissimo, il controllo di Bologna appunto, per l'espansione dell'egemonia viscontea. 
molte argomentazioni affastellate per indurre Carlo IV a scendere in Italia, viene ribadito come la dimora dei Cesari, la loro vera patria, sia comunque e necessariamente Roma ${ }^{50}$. Roma, riecheggiando il testo autorevole romano giustinianeo nonché la scienza civilistica medievale, è "communis patria" di tutti i sudditi dell'impero, sorta di sintesi e di luogo naturale del potere imperiale ${ }^{51}$. Roma è la regina delle città e del mondo, la capitale dell'impero. Proprio per questo, e particolarmente, vi è un legame strettissimo e intensissimo, perché necessario e inscindibile, tra i titolari della sovranità universale e la città di Roma.

A distanza, con ogni probabilità, di pochi mesi, ma in una situazione diversa quanto al rapporto tra Petrarca e Carlo, il tema della appartenenza reciproca tra Roma e l'Italia e l'imperatore si ripresenta. Nella lettera, scritta da Padova nel luglio del 1361, con cui Petrarca ringrazia Carlo IV del dono gradito e prestigioso di un cratere aureo, missiva del tutto aliena (né altrimenti poteva essere) da toni aspri e di rimprovero, ricorre l'invocazione a venire in Italia ${ }^{52}$, presumibilmente dettata da circostanze politico diplomatiche non distanti da quelle che avevano ispirato la lettera del marzo dello stesso anno ma orientate a analoghe esigenze della signoria carrarese. Tale ormai consueta esortazione viene enunciata, tra l'altro, in nome dell'"officium necessarium", del compito che inerisce all'essere stesso di chi incarna la sovranità impe-

Si tenga presente poi che in quegli stessi mesi una analoga direttrice di espansione, quella verso il Monferrato, conosceva uno stallo significativo. Il Marchesato si rivelava impenetrabile, nonostante i travagli politici interni che stava attraversando; forse solo un intervento del superiore feudale (il Monferrato è feudo imperiale) avrebbe potuto volgere la situazione in senso favorevole ai Visconti.

50 Fam. XXIII, 2 34: "Habet iam Boemia suum regem; tu Italie mundique rex, post tergum linquendi orbis iam securus, et patriam et solium tuum pete. Nam etsi secundum apostolicam sententiam manentem hic non habeas civitatem, siqua tamen in terris patria tua est, propria Cesarum domus ac vera patria Roma est; quin et comunis omnium est patria, rerum caput, orbis atque urbium regina”.

51 L. Roma, Ad municipalem (D. 50, 1, 33): "Roma communis patria est". Sul significato che tale massima poteva avere in Modestino, che l'ha formulata o sintetizzata, e nei giuristi dell'età imperiale cfr. F. Casavola, Il concetto di «Urbs Roma»»: Giuristi e imperatori Romani, Labeo 38 (1992), 20-29; una sintesi suggestiva del senso in cui l'intesero i giuristi medievali in E. H. Kantorowicz, The King's Two Bodies, Princeton, 1957, pp. 247-9.

52 Fam. XXIII, 8 5: "Quid inquam fiet, Cesar? Tu me in Germaniam, ego in Italiam te voco; tu auctoritate me superas, ego te causa; tu me ad delectationem honestam, non infitior, iocundamque, ego te ad virtutem atque officium arcesso necessarium ac debitum". 
riale. Non è quasi il caso di segnalare come "officium" sia tra i termini e concetti tematicamente più rilevanti nella filosofia ciceroniana dove, traducendo e rielaborando la media Stoà, indica il compito che appartiene a ogni uomo entro l'ordine cosmico. "Officium" è però anche un termine importante e significativo del linguaggio del diritto pubblico e non si può ritenere che Petrarca lo impieghi ingenuamente o in un senso che non sia quello tecnico suo proprio. Per una sintesi si può fare riferimento al primo vero dizionario giuridico, composto pochissimi anni prima da Alberico da Rosciate, per più di un verso vicino a Petrarca e agli ambienti che questi frequentava". "Officium" è la funzione dell" officialis", per eccellenza il "iudex", ossia il suo compito di enunciare il diritto e di restaurarlo dove è stato turbato; ma è anche il compito degli alti funzionari pubblici, dei vertici dell'amministrazione, dal momento che coincide con la "cura presidis prouincialis" ${ }^{54}$. La sovranità imperiale universale rappresenta certo il momento più alto di questa funzione fondamentale di garanzia dell'ordine giuridico. Non è possibile dire se Petrarca abbia tratto questo riferimento da una o dall'altra fonte, o da entrambe: infatti la matrice più schiettamente ciceroniana e quella civilistica sono perfettamente compatibili; il che non stupisce affatto solo che si ricordi quanto il diritto romano sia impregnato di filosofia ellenistica, stoica in particolare e ciò largamente in virtù della mediazione dell'arpinate. Rileva maggiormente il passaggio conclusivo. L'imperatore, per svolgere nel modo migliore, o meglio per svolgere davvero, quel compito altissimo di garante del diritto cui corrisponde anche la garanzia efficace dell'ordine del mondo, deve essere presente in quell'Italia che è, come si è visto, necessariamente la sede dell'impero.

Si può ricordare come l'inerenza reciproca tra l'Italia e l'imperatore appare da ultimo in toni più lirici, vicini al linguaggio degli affetti e delle emozioni, nella penultima Familiare indirizzata a Carlo IV, durante la dimora a Venezia l'11 marzo $1363^{55}$. La prosopopea dell'Italia delinea un vero e proprio rapporto amoroso con l'imperatore.

53 Si veda sempre G. Billanovich, Epitafio, libri e amici di Alberico da Rosciate, Italia medioevale e umanistica 3 (1961), pp. 252-61.

54 Cfr. Alberici de Rosate Dictionarium, ed. Bononie, 1 settembre 1481, s.v. Offitia.

55 Fam. XXIII 15, 6: "Clarum ac suave nomen est Cesar verendumque omnibus gentibus ac regibus, nobis vero etiam salutare; sine illud in nostro ore dulcescere, 
Come si è detto, il punto cruciale di queste argomentazioni di Petrarca, ossia che l'avere sede in Italia e a Roma inerisce alla dimensione ontologica dell'impero e che l'imperatore solo essendo romano può svolgere la pienezza delle sue funzioni di sovrano universale, costituisce un presupposto di tanta letteratura giuridica e pubblicistica. Se però si volesse indicare, tra i molti possibili, un riferimento preciso, si può pensare al giurista milanese Signorolo degli Omodei, attivo tra Milano e Pavia negli stessi anni in cui Petrarca era legato alla corte viscontea ${ }^{56}$ e che con ogni probabilità doveva essere noto al poeta, se non altro per l'intermediazione di Gabrio degli Zamorei57. Signorolo infatti presenta, nella lettura al Proemio del Digesto ${ }^{58}$, in modo particolarmente netto e sintetico questa esaltazione del ruolo imperiale di Roma, per cui solo nel legame con l'Urbe si ha la pienezza dei poteri e della sovranità universale.

Questo tema in Petrarca riecheggia il programma di restaurazione del ruolo imperiale esclusivo di Roma come è formulato nell'Epistola ai Romani di Manfredi di Svevia. Roma è per l'essenza sua e dell'impero la “mater imperiï", unica sede possibile dell'impero universale $e^{59}$. L' appartenenza reciproca tra Roma e l'imperatore viene delineata dalla cancelleria

seu illud clamantes seu taciti invocemus. Audi vel in silentio tuorum voces; tua te, Cesar, vocat Italia idque tantis clamoribus ut si auribus audiantur, non principem modo tuum, sed Indorum reges ultimos sint moturi; tua te, inquam, Italia Cesar vocat: 'Cesar, Cesar, Cesar meus, ubi es? cur me deseris? Quid cuntaris? Certe ego nisi immobilis forem, et dextra levaque mari gemino, a tergo autem alpibus circumsepta, ipsa iampridem meum Cesarem longe trans Danubium petisse"”.

56 A. Belloni, Signorolo degli Omodei e gli inizi della scuola giuridica pavese, Bullettino della Società pavese di storia patria 85 (1985), pp. 29-39.

57 Gabrio degli Zamorei, amico di Petrarca dagli anni di Parma, entra su sollecitazione di Galeazzo Visconti a fare parte del Collegio dei giuristi pavesi, cui attendeva anche Signorolo. Cfr. M.G. di Renzo Villata - G.P. Massetto, La Facoltà legale. L'insegnamento del diritto civile (1361-1535), in Almum Studium Papiense. Storia dell'Università di Pavia, vol. 1 t. 1, Milano, 2012, pp. 429-66, p. 438; C. Faraggiana di Sarzana, Gabrio Zamorei: un funzionario visconteo amico del Petrarca, Studi petrarcheschi 1 (1984), pp. 227-44.

58 Signorolo degli Omodei, lectura Proemii fforum, in Consilia questiones et allegationes, Mediolani 1497, cons. 129, segn. q iii v: "In civitate Rome imperator consequetur totalem perfectionem sue administrationis adeo quod nihil superest ad agendum. Et ideo ad denotandum perfectissimum esse ipsius impera. ibi recipit coronam preciosissime et perfectissime materiei idest aurea". Cfr. M. Cavina, Imperator romanorum triplici corona coronatur, Milano, 1991, pp. 107-12. urbs Roma”.

59 Manfredi di Svevia, Epistola ad Romanos cit., $\$ 17$, p. 224: "mater imperii 
di Manfredi ${ }^{60}$ nei termini di un rapporto naturale e generativo: Roma concepisce e dà alla luce il Cesare. Di conseguenza l'impero è romano proprio perché è allo stesso tempo universale: Roma è per l'impero come la radice per la pianta ${ }^{61}$. Questa dimensione per cui la natura stessa dell'impero chiarisce il ruolo imperiale di Roma non è mai venuta meno, quantunque possa avere conosciuto, e tuttora conosca nell'attualità, momenti di eclissi profonda. Il programma politico dichiarato qui da Manfredi di Svevia consiste per l'appunto nel restaurare a Roma la pienezza di questo suo ruolo eterno ${ }^{62}$. La permanenza, meglio il carattere immutabile, del ruolo imperiale di Roma trova il suo fondamento nel diritto. Il diritto di Roma non è mai davvero mutato, è ancora vigente, è l'unum ius per l'unum imperium; in virtù di questo diritto universale il potere imperiale di Roma antica è il potere imperiale della Roma attuale ${ }^{63}$.

Se si vuole cercare un contraltare polemico a queste posizioni di Petrarca e a lui prossimo, ci si può volgere a un brano di Oldrado da Ponte. Petrarca aveva certo avuto modo di conoscere bene il grande canonista lodigiano, sorta di consulente giuridico privilegiato dei papi lungamente attivo alla corte di Avignone $e^{64}$, e lo menziona anche nei

60 L'estensore, e in una certa misura anche coautore, dell'epistola dovrebbe essere il cancelliere di Manfredi, il notaio e giurista abruzzese Pietro da Prezze, su cui cfr. la voce Pietro da Prezza, dovuta a F. Delle Donne, in Dizionario biografico degli italiani, 83, Roma 2015.

61 Ivi, $\ 17$, p. 225 : "exurge quidem, o Roma, et intra claustra tui pectoris frequenti meditacione revolve, qualiter hactenus tuorum decurionum decreto, senatus auctoritate ac tui populi affirmacione in publico in sede imperii cesar electus... O radix felicissima invencionis imperii, primum concipiens Cesarem, Roma maxima, mundi caput, nunc capite cares".

62 Ivi, $\mathbb{S} 18$, p. 225: "Et ideo ex mansuetudinis serenitatis nostre tibi compacientes quam plurimum, te docere intendimus restitucionis in integrum titulum tibi modis omnibus opportunum, ut te Urbem inclitam, ad quam oculos maiestatis nostre dirigimus, solite tue dominacionis antiquo felicissimo statui restauremus... Hec tecum agere intendimus, Roma maxima, mundi capud, ut tam auctoritate tui senatus, populi et communis imperii iura resurgant et magestas nostri imperii solio preponatur, quam, imperiali nostra potencia privilegis renovatis, antiquissime tue sedis dominabilem gloriam reassumas, in qua olim singulis dominando tuorum imperatorum presidio duorum milium annorum curriculis et ultra, si bene recolis, presedisti".

63 Ivi, $\mathbb{S} 19$, p. 226: "Nec enim prefata sunt iuri contraria set consona racioni, cum, legum voluminibus exquisitis, intra sacrorum murorum tuum felicem circuitum illam eamdem hodie contineas potestatem, quam habuisti hactenus a primevo".

64 Per una ricostruzione complessiva e recente sia consentito il rinvio a $\mathrm{M}$. Conetti, Il testamento di Oldrado da Ponte (Avignone, 1334), in Quaderni di studi storici - Cahiers Adriana Petracchi 1 (2010), pp. 103-28. 
Rerum familiarium libri ${ }^{25}$. In un consilium di cruciale importanza politica, rivolto al papa in merito all'interdetto scagliato su Roma dopo le vicende legate all'incoronazione imperiale di Lodovico il Bavaro, Oldrado sviluppa una argomentazione del tutto diversa e contrapposta del primato di Roma, che appare fondato solo sul fatto che la chiesa romana è "mater et magistra" di tutte le altre chiese locali e della cattolicità nel suo insieme; configura una "translatio" della sede petrina da Antiochia a Roma, mimeticamente alla "translatio imperii"; afferma l'origine immediatamente divina della sede apostolica, in analogia a chi voleva rivendicare una analoga origine alla sovranità imperiale ${ }^{66}$. Oltre alla conoscenza, se non all'amicizia, diretta con Oldrado, un legame forte tra Petrarca e quelle vicende romane è costituito dal vero e grande amico del poeta, Jacopo Colonna il giovane. Come è noto fu il futuro vescovo di Lombez a leggere l'atto d'accusa pontificio contro Lodovico il Bavaro nella piazza di S. Marcello, l'aprile del $1328^{67}$.

\section{IL PAPA NON PUÒ IMPEDIRE ALL'IMPERATORE DI ESSERE ROMANO}

In una delle lettere a Neri Morando ${ }^{68}$ si trovano alcuni riferimenti,

65 Fam. IV 16, 13.

66 Oldrado da Ponte, Consilium 62, in Consilia et questiones, Roma 1472, senza segnatura ma p. 82 : "Sed pater sanctissime iura ista loquuntur in aliis ecclesiis et priuilegiis et non ecclesie romane et sic inter alias ciuitates et ciuitatem romanam magna est differentia. Illa enim est res publica alie loco priuatorum habentur ff de verborum significatione eum qui [D. 50, 16, 16], ad macedonianum 1. nihil [D. 14, 6, 17]. Ecclesia romana multis priuilegiis gaudet ... et est omnium mater et magistra $C$ de summa trinitate in epistola inter claras [C. 1, 2, 4]. Et ibi est fons sacerdotium... [p. 83 ] Illam enim imperator celestis instituit extra de electione fundamenta [rectius VI 1, 6, 17]. Ecclesia illa iubente domino ab antiochia in romam translata est xxiiii q. 1 rogamus [C. 24 q. 1 c.15]. Illa enim ipse solus fundauit et illius priuilegio fungitur xxii d. c. 1. Immo dicit textus quod non ab apostolis sed ab ipso saluatore nostro primatum obtinuit. Ipse enim locum elegit et uidetur quod etiam ratione persecutionis nolit eam deserere quod ex eo patet quia Petrum persecutionem fugientem ad illa reuocauit...". Su questo consilium cfr. C. Valsecchi, Oldrado da Ponte e i suoi Consilia. Un'auctoritas del primo Trecento, Milano, 2000, pp. 682-4; M. Conetti, Una sintesi di teoria e prassi: giuristi e potere nel primo Trecento, in Cecco d'Ascoli: cultura, scienza e politica nell'Italia del Trecento, atti del convegno Ascoli P. 2-3 dicembre 2005, Roma, 2007, pp. 301-17, pp. 315-6.

67 Basti il riferimento a G. Mollat, v. Colonna, Giacomo in Dictionnaire d'Histoire et Géographie Ecclesiastique, 13, 333.

68 Il libro XX delle Familiari si apre, come è noto, con due lettere all'amico 
incidentali rispetto all'insieme del discorso ma non per questo di scarso significato, al fatto che Carlo IV abbia lasciato con grande celerità l'Italia dopo avere ricevuto l'incoronazione imperiale ${ }^{69}$. Il fatto è macroscopico perché non è dipeso solo da una decisione dell'imperatore stesso, che avendo conseguito quello che doveva essere il suo scopo fondamentale non dimostra interesse a venire coinvolto nelle questioni romane e in genere nella politica toscana e lombarda. È stato soprattutto la conseguenza di un intervento diretto del papa, che avrebbe addirittura ordinato (Petrarca ricorre al verbo "iubeo") a Carlo di lasciare la città sede dell'impero. Alla luce dei temi visti sopra, e che si trovano qui riecheggiati, in questo modo il papa impedirebbe all'imperatore di svolgere le sue funzioni, svuoterebbe di contenuti la sovranità universale riducendola a una semplice definizione formale e esteriore per poteri di natura diversa, segnatamente quelli relativi al regno di Germania. Tale avvenimento viene citato assieme a altri esempi da Petrarca quale segno evidente della decadenza complessiva dei tempi presenti e in particolare delle difficoltà che vi incontra l'istituzione imperiale.

Lo stesso evento, coi significati di cui era carico, viene fatto oggetto di considerazioni più circostanziate nella Fam. XXIII $2^{70}$, come detto sopra particolarmente legata all'esigenza viscontea, nella primavera del 1361, di garantire il proprio progetto di potere mediante una presenza efficace dell'autorità imperiale, essendo venuta del tutto meno l'ipotesi di una collaborazione o se non altro di una non belligeranza da parte del papa e essendosi consolidata la resistenza da parte di alcuni poteri

forlivese; datate entrambe da Fracassetti al 1356, la XX 1 è stata anticipata da diversi studi (da ultimo E. H. Wilkins, Studies in the Life and Works of Petrarch, Cambridge (Mass.), 1958, pp. 103-4) al 1355. Per la lettera successiva che qui interessa si ha solo la proposta di datazione dovuta a H. Friedjung, Kaiser Karl IV. und sein Anteil am geistigen Leben seiner Zeit, Wien, 1876, p. 310, che insieme a Fam. XX 1 la data al 1355. Ai fini del discorso presente, la questione della data non rileva in modo particolare.

69 Fam. XX 2, 6: "iam Pontifex Romanus principem romanum Rome esse vetuerit, quod et fama loquitur et fuga Cesaris indicio est, qui non cupidus Italiam petiis quam reliquit, ut michi quidem supervacuo pelli videatur qui tam libens fugit"; Ivi 7: "Ut enim nunc intelligo, non hic aliam ob causam venit, nisi ut dyadema cesareum sua in sede susciperet; tantum adhuc reverentie, non amplius, superest. At successor Petri Cesaris successore securior, ista non curat, suumque ipse dyadema non pluris apud Tyberim quam apud Rodanum facit. Is nunc dyademate contentum et imperii titulo Roma digredi non solum patitur sed iubet, et quem imperatorem dici sinit, imperare autem nullo sit siturus modo".

70 Per il contesto della composizione cfr. supra, pp. 18-9. 
locali. Ecco allora la stigmatizzazione del comportamento, tenuto da Carlo IV allorchè lasciò Roma subito dopo avere ricevuta la corona imperiale, con cui avrebbe ottemperato alla promessa rafforzata da giuramento precedentemente formulata al $\mathrm{papa}^{71}$. Petrarca sottace che tale promessa avrebbe rappresentato una condizione necessaria per ottenere l'incoronazione imperiale. Ciò non solo poteva essere letto tra le righe del suo testo, era risaputo e aveva suscitato, come è noto, numerose polemiche; appare allora del tutto ovvio e comprensibile che Petrarca, in una missiva diretta proprio a Carlo, eviti di menzionare tale aspetto imbarazzante.

Petrarca, per quanto possa non essere immediatamente evidente, sviluppa il discorso facendo uso del linguaggio e dell'armamentario concettuale del diritto civile. Un simile impegno (la promessa giurata) si traduce sempre in una limitazione, sebbene volontaria e consapevole, per la libertà personale di chi lo contrae. Dal momento che il soggetto in questione è l'imperatore e che l'oggetto dell'impegno è, come si è visto in precedenza, un elemento costitutivo, essenziale, del potere imperiale, Carlo IV è giunto in questo modo a svuotare almeno in parte di significato la sovranità universale che di diritto gli compete. Proprio questo aspetto della promessa giurata da Carlo al papa, che la rende così sconvolgente sul piano della storia politica, permette però di individuare una via d'uscita sicura sul piano giuridico. Carlo IV infatti non era tenuto a ottemperarvi, pur avendola rafforzata col giuramento. Nel caso poi in cui, come in effetti è avvenuto, avesse comunque agito secondo quanto promesso, poteva senza alcun impedimento o difficoltà ritornare sui suoi passi, revocando le azioni compiute.

Dietro a questa argomentazione sviluppata da Petrarca sta un rinvio implicito ma decisivo alla scienza del diritto civile, che si era lungamente interrogata sui casi in cui il giuramento confirmatorio, che rafforza e conferma una promessa, o addirittura un contratto, poteva non essere

71 Fam. XXIII 2, 35: "Quas vero nunc more causas aucupabere quibusve deinceps te compedibus tentum dices? Nescio quid Romano pontifici pollicitus, iureiurando interposito, quasi muro valido seu monte invio, romane urbis aditu prohiberis; sic et summo principi suum imperium et summo imperio suus princeps, queque iacturarum omnium maxima est, tua tibi libertas eripitur. Sed iurasti, quod utinam non fecisses! Sed fecisti; dispensatione opus est. 38 Nunc vero, quenam ista superbia est, principem romanum publice libertatis auctorem libertate privare, ut cuius esse debent omnia, ipse non sit suus?”. 
valido. Si tratta di un problema estremamente complesso, sia per la pluralità di situazioni e fattispecie diverse che coinvolge, sia perché chiama in causa (vista la natura etica e religiosa del giuramento) intricate questioni di rapporti tra il diritto e la teologia, come pure tra giurisdizioni diverse, investendo anche il foro della coscienza e pertanto la giurisdizione ecclesiastica e penitenziale, con le competenze disciplinari specifiche del diritto canonico. A ogni modo, appare progressivamente chiaro e condiviso che in alcune situazioni il giuramento confirmatorio dovesse essere invalido o nullo. Questo avviene allorchè il giuramento sia legato a una obbligazione, anche contrattuale, che comporta una lesione enorme per chi lo presta, come ad esempio una compravendita a meno della metà del giusto prezzo ${ }^{72}$; oppure qualora i contenuti dell'obbligazione siano contra o praeter legem, senza però che i contraenti volessero con questo commettere un illecito ${ }^{73}$. Dopo dibattiti che avevano occupato generazioni, la civilistica bolognese, nell'età in cui Petrarca ivi attendeva agli studi, aveva sviluppato con ampiezza e sensibilità i casi di invalidità del giuramento confirmatorio $^{74}$. Si può pertanto ritenere che un'eco di questi anni di formazione sia qui viva e attiva. Volendo individuare un riferimento preciso, va vista senza dubbio l'affermazione netta di un maestro di Petrarca, molto legato inoltre ai figli di Stefano Colonna il vecchio, che erano suoi scolari, Jacopo e Agapito Colonna ${ }^{75}$. Si intende Jacopo da Belviso ${ }^{76}$, che

72 Cfr. le considerazioni sviluppate a partire dai glossatori, da Piacentino e Pillio da Medicina in particolare, sull'aut. Sacramenta puberum post C. 2, 28, 1. Per la ricostruzione di alcuni sviluppi successivi vissuti da questo tema come era stato impostato nell'età della glossa cfr. E. Cortese, La norma giuridica. Spunti teorici nel diritto comune classico, I, Milano, 1962, cap. 1 "La 'legge' di fronte al mondo etico-religioso", pp. 1-35, partic. pp. 1-9.

73 Cfr. la 1. Non dubium, De legibus et constitutionibus (C. 1, 14, 5).

$74 \mathrm{Si}$ vedano, quale esempio notevole di un orientamento dottrinale bolognese nel secondo quarto del XIV secolo, i commenti di Jacopo Bottrigari alla l. Non dubium (cfr. Iacobi Butrigarii Lectura super Codice; ed. Parrhisiis 1516, f. 29 vb - 31 rb) e all'aut. Sacramenta puberum (ed. cit., f. $74 \mathrm{vb}-76 \mathrm{ra}$ ).

75 Come si evince dal proemio della Lectura autenticorum cit. infra, f. $1 \mathrm{ra}$; cfr. D. Maffei, Giuristi medievali e falsificazioni editoriali del primo Cinquecento, Frankfurt am M., 1979, app. VII "Francesco Petrarca, tre scolari del Belviso e la data di composizione della Lectura Authenticorum", pp. 84-9.

76 Petrarca non menziona mai di essere stato allievo di Jacopo da Belviso; pure la cosa è più che plausibile, quasi certa, secondo la ricostruzione di F. Lo Parco, Francesco Petrarca e Tommaso Caloiro all'Università di Bologna, Studi e memorie per la storia dell'Università di Bologna 11 (1933), pp. 25-181, alle pp. 110 e ss. 
giunge a scrivere in modo lapidario: "iusiurandum illicitum servandum non est" $"$. Appare chiaro come, data la concezione del rapporto tra Roma e l'imperatore elaborata da Petrarca, il giuramento con cui l'imperatore stesso conferma la promessa di lasciare Roma si pone senz'altro con le caratteristiche dell'illiceità. All'imperatore infatti non può essere lecito spezzare, con la propria volontà, il rapporto che lo lega a Roma; il che evidentemente fulmina di nullità gli atti che vadano in questa direzione e libera dall'impegno a ottemperarvi.

Nel brano esaminato appena sopra, Petrarca affronta lo stesso problema e propone la stessa soluzione quali si trovavano in un testo giuridico legato a Arrigo VII e alla sua impresa italiana. Si tratta probabilmente di una sorta di parere da lui richiesto a uno, non meglio precisabile, dei giuristi che si trovavano alla sua corte in Italia, riguardo a varie questioni, nel complesso inerenti al sistema dottrinale di rapporti tra le due istanze universali, papato e impero, e legate a problemi che stavano travagliando la prassi politica. Tra queste, emerge l'interrogativo se al papa competa il potere di allontanare da Roma l'imperatore ${ }^{78}$. La risposta è, come era

77 Ad aut. Scenicas, $\mathbb{S}$ Novimus pridem, in Jacobi de Belviso Lectura autenticorum; ed. Lugduni 18 novembre 1511, f. 36 vb.

78 MGH Const. 4/2 app. VII, nr. 1248 (Memoriale imperatori porrectum), pp. 1308-17, pp. 1314-5: "An papa potuerit licenciare ab Urbe imperatorem, quia cum Roma sit caput imperii et de imperio et ex qua nomen accipi imperator, quia dicitur princeps Romanus, sequitur necessario quod eum inde abire iubere non potuit. Set huic dicto fortiter obloquitur XXIII q. VIII igitur [C. 23 q. 8 c. 7] ubi dicitur 'pro quo nostrum congregari precepimus populum'. Ubi glosator dicit et coligit, quod civitas romana est apostolici non imperatoris, nam imperator concessit ei civitatem cum ducato suo, ut LXIII d. Ego [D. 63 c. 1]. Set imperator est tantum ibi advocatus et defensor ecclesie, ut in dicto c. Extra de electione c. venerabilem [X 1, 6, 34]. Set ad hoc est parata responsio, quia translacio illa Lodoyci, que nititur ex translactione Constantini imperatoris, ut ibi dicit glosa, non tenuit, ut dicunt doctores in iure civili in dicta Autentica quomodo oporteat episcopos. Qua ratione illam Constantini passa est ecclesia magnificum Karolum dicere et scribere, quod habebat Romanos sibi subiectos ut et alias naciones, ut in dicto c. hec si quis XI q. I [C. 11 q. 1 c. 36] et imperator Iustinianus, qui post dictam translactionem Constantini fuit imperator, intitulat se principem Romanorum in prohemio Institutionum"; ivi, p. 1316: "dico, quod papa non potuit aliquibus condicionibus suspendere coronactionem imperatoris. Nam etsi etiam confirmactio imperatoris spectaret ad eum, non tamen est de actu vel ministerio confirmatis sicut nec eligentis adicere condiciones vel modos... Alioquin dicemus, quod non est imperator nisi in eventum vel defectum condicionis, quod penitus est falsum, vel si sub modo non impleto modo revocaretur imperium vel cogeretur implere modum, ut et lex dicit $\mathrm{C}$. de hiis que sub modo [C. 6,45$]$ et ff. de condictione ob causam [D. 12, 5] fere per totum". 
ovvio aspettarsi, negativa: al papa non può competere di allontanare l'imperatore da Roma, e questo in virtù del legame costitutivo tra il titolare della sovranità universale e il luogo ove questa risiede. Roma è il capo dell'impero, Roma inerisce all'impero cui fornisce il nome; a sua volta l'imperatore in quanto tale è romano, è il primo dei cittadini romani. La donazione di Costantino non ha valso a spezzare tali legami. L'anonimo autore del memoriale sembra essere convinto, sulla scorta in particolare di Accursio e della sua glossa Conferens generi, che la donazione sia invalida ${ }^{79}$. Anche però ammettendone l'ipotetica validità, questa non può in alcun modo mutare l'essenza dell'impero, che è pur sempre inscindibilmente legata a Roma. Ne consegue necessariamente che questo legame in quanto costitutivo per l'impero stesso non può in alcun modo spezzarsi: nessuna istanza di potere è dotata dell'autorità per ordinare all'imperatore di allontanarsi da Roma; pertanto gli eventuali impegni da lui assunti in proposito sono nulli e non producono alcun effetto. Si tratta di un testo poco noto e niente affatto studiato. Non stupisce allora che mai sia stato messo in relazione con queste particolari posizioni avanzate da Petrarca, nonostante l'assoluta identità ${ }^{80}$, che difficilmente potrebbe essere spiegata come una coincidenza casuale. Avremmo qui un'ulteriore e molto significativa continuità tra Petrarca e l'ambiente di Arrigo VII, la sua corte, il suo entourage politico e culturale durante il periodo italiano e toscano in particolare.

\section{L'IMPERATORE PORTA LA PACE}

La missione imperiale, lo scopo in vista del quale devono organizzarsi le attività e le funzioni dell'imperatore, consiste nell'assicurare il buon ordine e nel riportare la pace quando e dove questa fosse stata turbata.

La prima lettera a Carlo IV $^{81}$ gli rappresenta l'impero come da

79 Sia consentito il rinvio a M. Conetti, L'origine del potere legittimo. Spunti polemici contro la donazione di Costantino da Graziano a Lorenzo Valla, Parma, 2003, in partic. pp. 33-55.

80 Per quanto, come è ovvio, i due testi si esprimano in linguaggi diversi, facendo il memoriale uso dei procedimenti tecnico formali della scienza giuridica.

81 Fam. X, 15: "Romanum imperium multis diu iactatum tempestatibus sepe deluam et pene iam proiectam spem salutis in tua tandem virtute reposuit, et post innumeros casus sub umbraculo tui nominis utcunque respirat; sed sola in aspe diutius pasci nequit”. 
lungo tempo turbato e senza pace, proprio per questo tanto più fremente nell'attesa di un intervento efficace da parte del sovrano universale. La terza lettera riprende dalla prima e sviluppa anche questo tema, e lo fa in riferimento alla categoria di "officium" dell'imperatore ${ }^{82}$ : che consiste essenzialmente nel restaurare la pace e la giustizia ${ }^{83}$, ora profondamente ma non irrimediabilmente compromesse proprio a causa della latitanza dei poteri imperiali.

Il tema della giustizia e della pace è senza dubbio un luogo comune per la cultura politica di quelle generazioni, in relazione al potere imperiale, al definirne e esaltarne le funzioni ${ }^{84}$. Si risente qui ancora l'eco di numerosi documenti pubblici di Arrigo VII; ad esempio il diploma legato a uno dei primi grandi interventi, la costituzione di un vicario imperiale per la Lombardia ${ }^{85}$. Si concentra sulla descrizione del potere imperiale quale "officium", anzi "officium debitum" cui chi detiene la sovranità imperiale è tenuto a ottemperare, altrimenti dovrà renderne conto in ultima istanza davanti al giudizio divino. L'essenza di tale compito istituzionale sta proprio nel-riportare giustizia e pace dove sono state turbate. Turbamento che è proprio la conseguenza diretta dell'assenza stessa del potere imperiale, e si traduce in una mancanza di giustizia e di tranquillità. Ora invece il potere imperiale vuole essere presente e intervenire direttamente nella vita politica dell'Italia setten-

82 Per cui cfr. supra, p. 20

83 Fam. XVIII, 1 36: "Dirupta est, inquis, imperii libertas; tu pater imperii diruptam restauraberis; sumpta latinis servitus: tu illam tuorum cervicibus excuties; ad avaritie lupanar prostituta iustitia: tu illam ad sua sacra penetralia revocabis; pax e mentibus lapsa mortalium: tu illam in sua sede restitues. Ad hoc enim natus, huic officio destinatus es ut reipublice deformitates aboleas et pristinam faciem mundo reddas; tunc verus michi Cesar verusque videberis imperator, cum officium tuum impleveris".

84 Cfr. La pace nel pensiero, nella politica, negli ideali del Trecento. Atti del Convegno, Todi 13-16 ottobre 1974, Todi, 1975.

85 Nova Alamanniae, hrsg. von E.E. Stengel, I, Berlin, 1921, nr. 81, p. 38-9, p. 38: "Mailand 1311 um Januar 14 König Heinrich VII. verkündet den lombardischen Behörden die Ernennung des Grafens Amadeus von Savoyen zum Reichsvikar in der Lombardei: 'Attendentes igitur, quod ex regalis officii nostri debito, quod ex celesti dispensacione suscepimus, vilicacionis nostre rationem in die districti iudicii reddituri et qualitate diversorum negotiorum nostrorum et imperii per nonnullas et diversas partes maiestatis nostre presentiam necessario requirente in hiis partibus morari continuo principaliter nequeamus, ne maiestatis nostre absencia iusticia careatis, ecce pro exhibenda cuilibet sine difficultate iusticia, in qua sumus cuiuslibet debitores et pro recuperacione et defensione nostrorum et imperii iurium et honorum et conservacione boni et pacifici status vestri et totius Lombardie status ac dura gwerra nostris rebellibus inferenda...'”. 
trionale: questa restaurazione dei diritti dell'impero coincide con la conservazione della pace.

Lo stesso tema dell" officium" imperiale riemerge in un documento legato a una fase successiva dell'intervento di Arrigo VII in Italia. La convocazione del parlamento e dell'esercito, del dicembre $1312^{86}$, dove rispetto a un anno e mezzo prima e pure nel linguaggio formalizzato della cancelleria imperiale sembra di potere già scorgere toni quasi di sconforto, di fronte al perdurare di quello stato di violenza politica endemica che si manifesta anche come ribellione costante alla sacralità del potere imperiale. Viene a ogni modo ribadito che l'"officium" di chi detiene la sovranità imperiale si traduce sempre, né può essere altrimenti, nell'impegno a riportare la quiete, la pace.

Il sintagma "giustizia - pace" ricorre in un altro documento pubblico molto rilevante, la sentenza contro le città ribelli dell'aprile $1312^{87}$. Il prologo individua la preoccupazione fondamentale, che ispira l'intervento di Arrigo VII contro determinate città e le relative istituzioni pubbliche, nella necesità di sedare le violenze, estirpando le radici della discordia politica. Il potere imperiale consiste nell'assicurare giustizia e pace. Così i documenti dell'autunno $1312^{88}$, legati ai tentativi di pacificare le città toscane, riportano sovente il richiamo congiunto alla giustizia e alla pace.

In questo modo la comprensione che Petrarca esibisce di quello che è il compito fondamentale insito nell'esercizio del potere imperiale, coincide con la comprensione che ne dichiarano i testi ufficiali di Arrigo VII, in modo da potere difficilmente essere una coincidenza casuale.

86 MGH Const. 4/2, nr. 893 (Mandatum fidelibus missum de boste facienda et de parlamento convocando 18 dec. 1312), pp. 906-8, p. $907 \$ 1$ : "Levantes in circuitu oculos nostros et videntes guerrarum turbines, dissensiones et scandala, quibus humani generis inimicis sacrum imperium et subditos sibi populos per varias mundi partes turbat et vexat, post diversas nostre dilgentie curas, que pro ipsorum procuranda quiete ac optate pacis comodis promovendis, prout ad cesaree maiestatis officium pertinet...".

87 MGH Const. 4 / 2, nr. 768 (Sententia contra Lucam, Senas, Parmam, Regium, 1312 Apr. 11), pp. $757-63$, p. $758 \$ 1$ [prologo]; "A clamationibus etiam et a lacrimationibus multorum ex ipsis terris exulum nostra excitabatur intentio mensque regalis et animus cura vigili non cessabant, ut universam prefatam Tusciam dictis exstirpatis erroribus et discordiis ad pacis federa restaurare possemus et universis de Ytalia nostris et romani imperii fidelibus pacis et unitatis federe congaudentibus, et iusticie constantis exibitione Romanum fulgeret imperium et regalis maiestas as sacrarum susceptionem infularum Romam progredi”.

88 cfr. MGH Const. 4/2 nrr. 851-70, pp. 858-86. 


\section{IL FAVORE DELLA SITUAZIONE CONTINGENTE}

La retorica con i richiami costanti all'antichità classica, o meglio con la lettura sistematica dell'attualità anche politica nei termini della romanità imperiale, non deve offuscare il lettore inducendolo a pensare che Petrarca sia più attento alla storiografia di Tito Livio che all'apprezzamento dell'attualità politica o magari che quest'ultima gli interessi solo in quanto può essere reinterpretata in chiave classicheggiante. Un elemento molto importante delle esortazioni all'imperatore perché venga in Italia è costituito sempre da un apprezzamento accurato della situazione politica contingente. Petrarca si preoccupa di rappresentare a Carlo IV le condizioni particolarmente favorevoli per un'impresa cisalpina.

Nella prima lettera viene istituito il confronto, implicito e poi in chiusura reso esplicito, con precedenti discese imperiali e segnatamente quella del nonno Arrigo VII (ma Petrarca poteva avere in mente anche la politica italiana, assimilabile del resto in larga misura a quella di un imperatore, del padre di Carlo, Giovanni di Boemia, cui Carlo stesso giovinetto aveva preso parte). A fronte di quelle esperienze, recenti ma comunque consegnate al passato, al momento attuale non si avrebbe in Italia alcuna seria opposizione a una presenza vera e efficace del potere imperiale ${ }^{89}$. Nel prosieguo, è opportunamente la voce di Roma stessa a richiamare l'attenzione di Carlo IV sul fatto che persino i papi non sono mai stati tanto ben disposti quanto l'attuale a accettare la presenza dell'imperatore in Italia ${ }^{90}$; con un gioco di parole sul nome del pontefice regnante, Clemente di nome e di fatto "clemens" quanto mai altri predecessori verso il potere imperiale e chi lo deteneva. Può essere importante fare notare come tale gioco di parole ha un antecedente in un testo importante uscito dalla cancelleria di Arrigo VII ${ }^{91}$.

89 Fam. X 1, 10: "Adde quod nullius unquam externi principis adventum letius expectavit Italia ut que nec aliunde remedium vulneribus suis sperat nec tuum tanquam alienigene iugum timet. Hoc singulare, si nescis, habet apud nos maiestas tua...nobis post tot secula mos patrius et Augustus noster est redditus; te enim utlibet sibi Germani vendicent, nos te italicum arbitramur".

90 Ivi, $\mathbb{\$} 20$ : "Tu michi prope iam desperanti divinitus destinatus, quid cessas, quid cogitas, quid expectas? Nunquam aut ego tui egentior aut tu ad opem ferendam aptior aut Romanus pontifex clementior aut favor Dei et hominum propensior aut illustrior res agenda”.

91 MGH Const. 4/2, nr. 801 (Litterae encylcicae imperatoris Iun. 29 1312, 
La seconda missiva che Petrarca invia all'imperatore rappresenta come è noto una ripresa o una sintesi dei temi elaborati in quella precedente e inaugurale. Così anche per l'apprezzamento della contingenza politica. Addirittura le condizioni favorevoli vengono attribuite a un intervento della divinità, che con la sua provvidenza avrebbe disposto in tale modo la situazione italiana, da fare sì che proprio quello fosse il momento migliore possibile. Come tale andrebbe colto, giocando a favore di Carlo anche il sentimento diffuso di attesa per l'azione pacificatrice dell'impero, che rischia invece di raffreddarsi presto qualora non trovi pronta corrispondenza nell'impegno concreto dell'imperatore ${ }^{92}$. Non è chiaro se si tratti qui di un semplice espediente retorico volto a spezzare le resistenze di Carlo convincendolo a approfittare del momento; oppure se davvero Petrarca fosse convinto che difficilmente si sarebbero potute presentare di nuovo condizioni tanto propizie.

In tale prospettiva aiuterebbe potere comprendere a quali elementi in particolare dell'attualità politica Petrarca stava pensando, ossia di quale attualità si tratti, a quando esattamente datare la lettera. Il poeta non vi ha posto alcuna indicazione cronica. Una convinzione comune della critica la data al 1352 , meglio ai primi mesi33; è stato proposto però di anticiparla al $1351^{94}$. Poco oltre nella stessa missiva Petrarca fa un rife-

Encyclica in forma maiori), pp. 801-4, $\mathbb{2}$ 2, p. 803, dove si fa riferimento all'innata "clementia" di papa Clemente, allora ovviamente il V del nome laddove la Familiare in questione allude al VI.

92 Fam. XII 1, 2: "Nunc tandem preparante divina providentia vias tuas et fidelis populi salutem misericorditer ordinante, ita dispositus est Italie tue status adeoque fervens expectatio, ut dilationem ipsam que gloriosis primordiis intercessit, eidem providentie ascribam potiusquam fortune, quo scilicet immensum exoptatissimi principis mora brevis ardorem publicum excitaret. Iam vero bonorum animos ardere amplius non posse tibi persuadeas velim verendumque ne, quod natura rerum fert, incendio sensim tepor obrepat nisi generosis flammis, quas tuum nomen accendit, presentie tue alimenta perbueris".

93 A partire da Fracassetti fino a Wilkins, Studies cit. p. 107 n. 38, passando per A. Jäger, Francesco Petrarcas Brief an Kaiser Karl IV. über das österreichische Privilegium vom jahre 1058, Archiv für österreichische Geschichte 38 (1867), p. 449; Piur, Petrarcas Briefwechsel cit. pp. 7-8; C.C. Bayley, Petrarch, Charles IV and the Renovatio Imperii, Speculum 17 (1942), a p. 329.

94 G. Pirchan, Die Abfassungszeit der ersten drei Briefe Petrarcas an König Karl, Mitteilungen der Österreichisches Institut für Geschichtsforschung, Ergänzungsband 11 (1929), pp. 374-85. 
rimento più preciso alla situazione in Toscan $a^{95}$; certo poiché gli sconvolgimenti e le violenze politiche diffuse in quell'area avevano determinato tanto lo scacco della politica imperiale di Arrigo VII quanto nel 1332 la fine della signoria del padre Giovanni di Boemia su Lucca, di cui Carlo era vicario benchè giovanissimo. Proprio quella Toscana e quella Firenze che si erano dimostrate esiziali alla casa di Lussemburgo, al presente appaiono pronte a ossequiare il re dei romani.

Questa allusione a una congiuntura della politica toscana particolarmente favorevole per gli interessi imperiali potrebbe richiamare una fase delle ostilità tra i Visconti e Firenze. Determinate dall'espansione viscontea a Bologna, allorchè Giovanni Visconti da Oleggio vi è nominato Capitano del Popolo, nell'aprile 1351, iniziano nell'estate dello stesso anno con gli assedi viscontei a Prato e Pistoia e conoscono il momento più intenso e di maggiore pericolo per Firenze con l'offensiva milanese della primavera 1352. Proprio questa situazione di minaccia profonda e immediata induce Firenze a cercare l'appoggio imperiale, tanto che un primo accordo con Carlo IV viene siglato il 30 aprile.

Nella primavera del 1352 Firenze, che era sempre stata l'anima delle opposizioni anti imperiali in Toscana, ha cercato e ottenuto l'accordo con l'imperatore. Si può pensare che Petrarca alluda precisamente a tale circostanza e seguire pertanto l'indicazione ${ }^{96}$, finora isolata, a datare la lettera in questione a aprile o maggio di quell'anno.

Si trova una risposta all'interrogativo: l'esortazione che Petrarca rivolge a Carlo IV, per indurlo a intervenire rapidamente traendo così vantaggio da una congiuntura tanto favorevole quanto inaudita e forse passeggera ${ }^{97}$ è tutt'altro che un vuoto esercizio di retorica. Al contrario nasce da una conoscenza approfondita della situazione politica e delle attività diplomatiche, che del resto vedevano coinvolta anche la corte papale. Petrarca è tutto dentro alle dinamiche della

95 Fam. XII 1, 8: "Intelligis, prudentissime princeps, non tantum quid loquar, sed quid cogitem; presentem Tuscie statum vides: ubi plurimum rebellionis avo tuo aliisque retro principibus fuit, illic tibi plurimum erit obsequii. Utere rerum mutatione felicissima sortemque tua ne neglexeris".

96 K. Palm, Italienische Ereignisse in den ersten jabre Karls IV., Göttingen, 1873 , pp. $58-60$.

97 Chi conosceva i sentimenti anti imperiali ormai consolidati a Firenze, e nessuno poteva conoscerli meglio di chi li aveva scontati già nascendo in esilio, poteva ritenere che l'accordo con Carlo IV sarebbe stato di breve durata. 
grande politica italiana e europea, che conosce e che sa valutare nei loro equilibri mutevoli.

Così il richiamo alla tradizione del ghibellinismo, di Manfredi e soprattutto di Arrigo VII, non è un compiacimento antiquariale. Acquisisce senso nel momento presente: si veda l'importante richiamo "dies est" gramma imperiale di Arrigo VII può in quel particolare momento essere ripreso con buone possibilità di successo.

Impossibile e inutile precisare se si tratti di una persuasione sincera o di una argomentazione volta a provocare l'intervento di Carlo IV funzionalmente a disegni e esigenze dei poteri cui Petrarca era legato quando gli scriveva. Importa porre in evidenza la sensibilità di Petrarca per le condizioni contingenti, il legame stretto con l'attualità, che non può non coinvolgere tutti i discorsi sull'impero e su Roma sviluppati pur sempre nella prospettiva di stimolare una politica italiana dell'imperatore.

Per la persistenza e l'efficacia del modello che Petrarca svolge in relazione alla figura del primo imperatore della casa di Lussemburgo, si tenga conto che Giovanni Boccaccio riprende gli elogi di Petrarca a Arrigo VII" .

\section{PER CONCLUDERE}

Una volta mostrate, come auspicabilmente dovrebbe essere avvenuto nelle pagine precedenti, le parentele, ancor che in certi casi lontane, che connettono i testi di Petrarca alla tradizione pubblicistica e alla documentazione di Manfredi di Svevia e soprattutto di Arrigo VII, si tratta di provare a sostenere che effettivamente, in virtù di concrete situazioni biografiche, Petrarca poteva avervi accesso.

Non mancano i legami che potevano rinviare Petrarca agli anni di Arrigo VII. I Colonna, in particolare Stefano il Vecchio ${ }^{100}$, cui Petrarca

98 Fam. XXIII 15, 6.

99 Come segnalato da A. Hortis, Studi sulle opere latine del Boccaccio, Trieste, 1881, p. 151 n. 6 e da G. Billanovich, Petrarca letterato, I Lo scrittoio del Petrarca, Roma, 1947, p. 120.

100 Ricorre spesso nei documenti pubblici di Arrigo VII, specialmente quelli legati alle vicende dell'incoronazione romana. 
tanto e tanto a lungo rimase legato, erano molto prossimi a Arrigo VII nel periodo italiano. Il legame più intenso potrebbe però essere rappresentato proprio dal padre. Ser Pietro, meglio noto come Petracco, di Parenzo, dapprima nel 1304 trova momentaneo rifugio a Arezzo (dove nasce Francesco), centro anti fiorentino e filo imperiale che di lì a pochi anni avrebbe aderito integralmente al programma di Arrigo. Soprattutto ser Petracco si trasferisce a Pisa nel 1311, nel luogo che costituiva il centro della politica imperiale di Arrigo VII in Toscana e proprio col fine di legarsi strettamente a lui e alla sua corte. Solo quando intuisce che la sua politica è destinata a fallire o per lo meno che non riuscirà a consentire ai banditi di rientrare serenamente a Firenze, giunge la scelta di abbandonare la Toscana per Avignone.

Si spiega facilmente l'assenza di riferimenti a Lodovico il Bavaro e alla pubblicistica di parte imperiale che la sua vicenda politica ha suscitato, per quanto potessero risultare utili a corroborare alcune idee presentate da Petrarca. Si trattava infatti di una posizione estranea se non ostile a quella tenuta complessivamente dai Colonna. Inoltre e forse a maggior ragione, la casa di Lussemburgo e quella di Baviera erano nemiche acerrime, in virtù della competizione per il trono imperiale e ancora più della causa matrimoniale legata alla contessa del Tirolo, Margherita Maultasch, e al controllo della cruciale regione alpina. Per quanto allora Petrarca potesse essere rimasto accattivato dall'unica incoronazione imperiale compiuta non dal papa ma dal popolo di Roma, si trattava di un esempio da evitare assolutamente scrivendo a Carlo IV.

La lettera di Manfredi di Svevia ai romani dovette essere oggetto di una sorta di damnatio memoriae ${ }^{101}$; pure se ne è ipotizzata una lunga e efficace sopravvivenza, segnatamente in Cola di Rienzo ${ }^{102}$. Se così fosse, si potrebbe pensare a Cola e all'ambiente romano come al tramite che l'ha resa nota a Petrarca.

Non si vuole affermare che Petrarca avesse una conoscenza dettagliata di quanto era uscito dalla cancelleria di Arrigo VII (e eventualmente di Manfredi di Svevia). Salvo forse in alcuni casi, per alcuni testi

101 È tradita da un manoscritto unico, il c.d. Codice Fitalia (Palermo, Biblioteca della Società siciliana per la storia patria, I B 25) che ne presenta una trascrizione imprecisa; cfr. nota 36.

102 K. Burdach, Briefwechsel des Cola di Rienzo, Berlin, 1912-1929, ad indicem. 
meglio noti e che avevano effettivamente goduto di una maggiore diffusione, quali l'enciclica di Arrigo VII per l'incoronazione e il memoriale presentatogli in materia di rapporti col papato, forse anche l'epistola ai romani di Manfredi; testi che Petrarca potrebbe avere conosciuto e quindi citare. Altrimenti, si tratta piuttosto di eco e reminiscenze, rivelate dalla consonanza di idee e linguaggio, dovute al fatto che tutto un armamentario di idee e di linguaggio poteva essersi conservato, nell'ambiente familiare di Petrarca come pure in quello colonnese.

Oltre e accanto ai documenti pubblici e ai pamphlet legati a Manfredi di Svevia e Arrigo VII; si segnala una presenza occasionale ma non incidentale della scienza del diritto civile. Si tratta di considerazioni legate al diritto pubblico imperiale o a rapporti privatistici ma applicate (come del resto avveniva sovente) a problemi politici; aiutano Petrarca a definire meglio i profili della sovranità imperiale e il sistema di rapporti con la suprema autorità spirituale. Sono con ogni probabilità sia reminiscenze degli anni bolognesi sia eco della civilistica pavese nel periodo in cui si trovava alla corte viscontea. Anche in questo caso non abbiamo citazioni letterali, non vengono riprese alla lettera linee argomentative. Si segnala piuttosto una sorta di consonanza, per cui Petrarca non manca, qualora gli appaia evidentemente opportuno o utile, fare appello anche alle competenze giuridiche di cui si doveva essere dotato negli anni di studio, per inserirle strumentalmente entro i vari discorsi.

L'elaborazione di una teoria politica, come si diceva in esordio, è assente da questi brani di Petrarca: non vi si può ravvisare, nemmeno in modo frammentario, una dottrina del potere imperiale. Al contrario, l'argomentare, anche quando vuole presentarsi quale visione d'insieme, è sempre legato alle contingenze dell'attualità immediata e degli scenari diplomatici cui Petrarca si trova connesso. Pure, almeno un concetto chiave sembra attraversare tutti questi momenti: l'idea imperiale di Roma, per riprendere la categoria interpretativa formulata a suo tempo da Eugenio Dupré Theseider ${ }^{103}$ onde leggere tanta parte della pubblicistica in particolare tra metà Duecento e metà Trecento. L'idea che l'impero romano non sia mai venuto meno, se non nella realtà effettuale dei

103 Idea che sintetizza così: "la persuasione... che l'alma città fosse tuttora, come nei favolosi tempi degli antichi Cesari, la fonte e sede dell'imperium per eccellenza, e, grazie a ciò, il centro del dominio del mondo" (Dupré, L'idea cit., p. 7). 
poteri, nella validità della sovranità universale. Soprattutto, il legame ontologico, pertanto inscindibile, tra l'impero universale e l'Italia in senso più ampio, Roma in senso specifico.

La centralità dell'idea di Roma per le visioni politiche di Petrarca è acquisizione risalente e pressochè unanime della critica. L'elemento di novità che questo contributo vorrebbe inserire, sta nel richiamare l'attenzione su due elementi notevoli.

In primo luogo, la natura ontologicamente romana della sovranità imperiale, quale si presenta nelle pagine dei Rerum familiarium libri, non costituisce affatto un'idea originale di Petrarca. Al contrario, deriva direttamente da testi e tradizioni bene individuabili, che si tratti della cancelleria imperiale o della scienza del diritto romano. Senza dubbio, questa idea viene formulata nel linguaggio particolarissimo di Petrarca, col rinvio costante all'antichità mediata dalla storiografia romana e col recupero di un modello linguistico volto a fare, come è stato detto, di Petrarca il Cicerone del suo tempo ${ }^{104}$. Quanto però ai contenuti di pensiero, non è dato ravvisare una elaborazione originale rispetto alle sue fonti.

In secondo luogo, va tenuto presente che nei Rerum familiarium libri l'idea imperiale di Roma compare quasi sempre strumentalmente alle esigenze politico diplomatiche contingenti sottese alle varie missive e nel legame con l'attualità politica. Si presenta dapprima quale un mezzo efficace con cui Petrarca ritiene di potersi accreditare positivamente presso i fronti in senso lato ghibellini, che si tratti di esponenti del collegio cardinalizio o dei Visconti; ricorre quale argomentazione volta assieme a altre al fine di determinare l'imperatore a svolgere una politica italiana.

Tali considerazioni potrebbero revocare in dubbio l'opportunità di considerare l'idea di Roma e dell'impero universale un punto fermo, forse l'unico vero concetto immutabile, entro le concezioni politiche di Petrarca; inducendo invece a considerarle uno tra $\mathrm{i}$ tanti espedienti strumentali di cui il poeta fa uso nelle varie situazioni particolari.-

Se è stato possibile ravvisare l'idea di Roma quale costante nei Rerum familiarium libri, ciò è forse dovuto proprio alla sapiente architettura con cui Petrarca ha costruito e assemblato il suo grande testo ${ }^{105}$.

104 Cfr. ad es. Martelli, Petrarca cit., p. xiv.

105 Per cui cfr. per lo meno Billanovich, Lo scrittoio cit., e in tempi recentissimi Antognini, Il progetto cit. 
L'analisi delle singole missive mostra Petrarca quale ghibellino d'occasione, per cui l'esaltazione dell'impero di Roma è strumentale a esigenze contingenti; si potrebbe però ipotizzare che, nei momenti in cui viene assemblando la raccolta delle lettere familiari Petrarca utilizzi anche questi riferimenti occasionali nel quadro di una esaltazione complessiva della romanità classica e delle sue persistenze, o della possibilità di recuperarla nel presente, con una operazione che però prescinde ormai in larga misura da significati di natura politica. 